\title{
A PRIORI ESTIMATES FOR A RELATIVISTIC LIQUID WITH FREE SURFACE BOUNDARY
}

\author{
DAN GINSBERG
}

\begin{abstract}
We prove energy estimates for a relativistic free liquid body with sufficiently small fluid velocity in a general Einstein spacetime. These estimates control Sobolev norms of the fluid velocity and enthalpy in the interior as well as Sobolev norms of the second fundamental form on the boundary.
\end{abstract}

\section{The Relativistic Euler Equations}

For a given globally hyperbolic spacetime $(M, g)$ we consider the motion of an isentropic relativistic perfect fluid:

$$
\begin{array}{ll}
\nabla_{u} u^{\mu}+\frac{1}{\epsilon+p} \Pi^{\mu \nu} \nabla_{\nu} p=0, & \text { in } \mathcal{D} \\
\nabla_{u} \rho+\rho \nabla_{\mu} u^{\mu}=0, & \text { in } \mathcal{D},
\end{array}
$$

for $\mu, \nu=0, . ., 3$. We are employing Einstein notation and summing over all repeated upper and lower indices. Here, $u$ is the four-velocity of the fluid, which is a unit timelike future-directed vector field. The variables $\epsilon, p, \rho$ are the energy density, pressure, and mass density, respectively, and are all assumed to be non-negative in $\mathcal{D}$, and $\Pi$ denotes the projection to the orthogonal complement of $u$ :

$$
\Pi^{\mu \nu}=g^{\mu \nu}+u^{\mu} u^{\nu} .
$$

We are writing $\nabla$ for the covariant derivative associated to $g$ and $\nabla_{u}=u^{\mu} \nabla_{\mu}$ and are working in units so that the speed of light is 1 . The set $\mathcal{D} \subset M$ is to be determined, and will satisfy the following constraints.

We suppose that $M$ is foliated by spacelike hypersurfaces $M_{t}$. Let $\mathcal{D}_{0}$ be a bounded domain diffeomorphic to the unit ball and contained in $M_{0}$. Given a velocity field $\left(u_{0}\right)^{\mu}$, energy density $\epsilon_{0}$, mass density $\rho_{0}$ and pressure $p_{0}$ on $\mathcal{D}_{0}$ satisfying:

$$
\begin{array}{ll}
\left(u_{0}\right)^{\mu}\left(u_{0}\right)_{\mu}=-1, & \text { in } \mathcal{D}_{0}, \\
\epsilon_{0}, \rho_{0}, p_{0}>0 & \text { in } \mathcal{D}_{0}, \\
\epsilon_{0}, \rho_{0}>0 & \text { on } \partial \mathcal{D}_{0}, \\
p_{0}=0 & \text { on } \partial \mathcal{D}_{0},
\end{array}
$$

we want the domain $\mathcal{D}=\bigcup_{0 \leq t \leq T}\{t\} \times \mathcal{D}_{t}$ to satisfy:

$$
\begin{array}{ll}
u^{\mu} u_{\mu}=-1 & \text { in } \mathcal{D}, \\
\epsilon, \rho, p>0 & \text { in } \mathcal{D}, \\
p=0 & \text { on } \Lambda, \\
u^{\mu} N_{\mu}=0 & \text { on } \Lambda,
\end{array}
$$

where $N_{\mu}$ is the unit conormal to $\Lambda=\bigcup_{0 \leq t \leq T}\{t\} \times \partial \mathcal{D}_{t}$.

In the non-relativistic setting, it is well known (see [1]) that the analog of the above problem is ill-posed unless the "Taylor sign condition" holds. This is the requirement that:

$$
\nabla_{N} p>-\delta>0 \text { on } \Lambda, \quad \text { where } \nabla_{N}=N^{i} \nabla_{i} .
$$

Date: November 20, 2018. 
The above equations do not close, because there are six independent variables but only four equations. For an isentropic fluid, the energy density $\epsilon$ is a function of the mass density $\rho, \epsilon=\epsilon(\rho)$ for some smooth, non-negative, increasing function $\epsilon$, which is determined from the other thermodynamical variables $\rho, p$ by solving:

$$
\frac{d \epsilon}{d \rho}=\frac{\epsilon+p}{\rho}
$$

We will also assume that the fluid is barotropic, which means that $p=p(\epsilon)$ for a given smooth, nonnegative increasing function $p$. In this case, (1.10) implies that $\epsilon \equiv \epsilon_{0}$ on the boundary and therefore $\rho=\rho_{0}$ on $\partial \mathcal{D}_{t}$ as well. The assumption that $\rho_{0}, \epsilon_{0}>0$ corresponds to an equation of state modeling a liquid.

Using (1.13), given any one of the variables $p, \rho, \epsilon$, we can determine the other two. In fact it is convenient to work in terms of the (specific) enthalpy $\sqrt{\sigma}$ :

$$
\sqrt{\sigma}=\frac{\epsilon+p}{\rho}
$$

If $p=p(\epsilon)$ is given, then both $\epsilon, \rho$ can be determined from (1.13) once $\sigma$ is known. From now on, we will think of $\sigma$ as the fundamental thermodynamical variable, so $(p, \rho, \epsilon)=(p(\sigma), \rho(\sigma), \epsilon(\sigma))$.

If we set

$$
V^{\mu}=\sqrt{\sigma} u^{\mu}
$$

then using (1.13), the equations (1.1) and (1.2) can be written in terms of $V$ and $\sigma$ :

$$
\begin{aligned}
& \nabla_{V} V^{\mu}+\frac{1}{2} g^{\mu \nu} \nabla_{\nu} \sigma=0, \\
& \nabla_{V} e(\sigma)+\nabla_{\mu} V^{\mu}=0,
\end{aligned}
$$

where $e(\sigma)=\log (\rho(\sigma) / \sqrt{\sigma})$, and the boundary conditions are:

$$
\begin{aligned}
\sigma & =\sigma_{0}, & & \text { on } \partial \mathcal{D}_{t}, \\
N_{\mu} V^{\mu} & =0, & & \text { on } \partial \mathcal{D}_{t},
\end{aligned}
$$

with $\sigma_{0}=\frac{\epsilon_{0}+p_{0}}{\rho_{0}}$. For a derivation of (1.16)-(1.17), see, for example [2]. We will derive energy estimates for the system (1.16)-(1.19) with sufficiently small fluid velocity, assuming that (1.12) holds.

1.1. Previous results. In the non-relativistic setting, if $\mathcal{D}_{t}$ is unbounded and the fluid is incompressible and irrotational, the above is known as the "water waves" problem. In this case, global existence for small initial data has been proven using a variety of techniques; see for example [3], 4] as well as the recent survey article [5] for a detailed history of that problem.

For an incompressible bounded fluid body with nonvanishing curl, in [6], Christodoulou-Lindblad prove energy estimates that control the fluid velocity and the second fundamental form of the boundary, and our approach is modeled on theirs. Local well-posedness for this case was first proved by Lindblad in [7] using a Nash-Moser iteration. Coutand-Shkoller [8] were able to avoid the use of Nash-Moser by using a tangential smoothing operator and fractional Sobolev spaces. In his PhD. thesis, Nordgren [9] modified the methods of [8] and proved local well-posedness for a self-gravitating incompressible fluid.

In the case of a compressible liquid body, local well-posedness was again proved by Lindblad in [10] using a Nash-Moser iteration. In [11, the authors showed local well-posedness by introducing an "artificial viscosity" term and studying the zero viscosity limit. See also the recent paper [12] where the estimates from [6] are extended to handle a compressible liquid. The case that $\rho=0$ on $\partial \mathcal{D}_{t}$ is known as a gas; see for example [13, for a priori estimates and [14, 15, 11] for local well-posedness. See also [16], where a nontrivial family of global-in-time solutions are constructed for a self-gravitating compressible gas.

Moving now to the free boundary problem for the relativistic Euler equations, the monographs [17, [18] and [19] contain a detailed analysis of shock formation in the exterior of an irrotational fluid body. For the interior of a liquid body with non-vanishing vorticity, Oliynyk [20] recently obtained energy estimates in in Lagrangian coordinates. The approach and estimates there are quite different from the present paper; Oliynyk estimates Lagrangian derivatives of the fluid velocity and the Lagrangian coordinate map, while we derive estimates for Eulerian derivatives of the velocity and the second fundamental form. We remark that the assumption (2.35) is not needed for the estimates in 20]; see the discussion after (2.35). Oliynyk also recently announced a proof of local well-posedness for this problem; see 21]. See also 22] and [23] for energy estimates for relativistic gas bodies. 
1.2. Energy estimates. We begin by recalling the basic energy associated to the system (1.1)-(1.2) with boundary conditions (1.10)-(1.11). The equations (1.1) are a consequence of the Einstein equations:

$$
R^{\mu \nu}-\frac{1}{2} R g^{\mu \nu}=T^{\mu \nu}
$$

where $T^{\mu \nu}$ is the energy-momentum tensor for a perfect fluid, defined by:

$$
T^{\mu \nu}=(\epsilon+p) u^{\mu} u^{\nu}+p g^{\mu \nu},
$$

and $R^{\mu \nu}, R$ are the Ricci and scalar curvatures of $g$, respectively. By the second Bianchi identity, $\nabla_{\mu} T^{\mu \nu}=0$, and (1.1) is then obtained by considering the component of (1.20) orthogonal to $u$ (see 24] or [25] for a derivation).

We let $\tau_{\mu}$ denote the future-directed unit normal to the time slices $\mathcal{D}_{t}$ and we let $\bar{g}$ denote the induced metric on $\mathcal{D}_{t}$ (see section 2.1). Integrating $\tau_{\nu} \nabla_{\mu} T^{\mu \nu}=0$ over $\mathcal{D}$ gives:

$$
\begin{aligned}
0=\int_{\mathcal{D}} \tau_{\nu} \nabla_{\mu} T^{\mu \nu} d V_{g} & \\
& =\int_{\mathcal{D}_{t}}\left(-\tau_{\nu} \tau_{\mu}\right) T^{\mu \nu} d V_{\bar{g}}-\int_{\mathcal{D}_{0}}\left(-\tau_{\nu} \tau_{\mu}\right) T^{\mu \nu} d V_{\bar{g}}+\int_{\Lambda} \tau_{\nu} N_{\mu} T^{\mu \nu} d V_{\gamma}-\int_{\mathcal{D}} \nabla_{\mu}\left(\tau_{\nu}\right) T^{\mu \nu} d V_{g} .
\end{aligned}
$$

Here, $d V_{\bar{g}}$ and $d V_{\gamma}$ denote the volume element on $\mathcal{D}_{t}$ and $\Lambda$, respectively. The boundary term is:

$$
\int_{\Lambda} T^{\mu \nu} N_{\mu} \tau_{\nu} d V_{\gamma}=\int_{\Lambda}\left((p+\rho)\left(u^{\mu} N_{\mu}\right)\left(u^{\nu} \tau_{\nu}\right)+p N^{\mu} \tau_{\mu}\right) d V_{\gamma}=0
$$

by (1.10) and (1.11).

Writing $u^{\tau}=u^{\mu} \tau_{\mu}$, we have:

$$
\int_{\mathcal{D}_{t}}(\epsilon+p)\left(u^{\tau}\right)^{2}+p\left(\tau^{\mu} \tau_{\mu}\right) d V_{\bar{g}}=\int_{\mathcal{D}_{t}} \epsilon\left(u^{\tau}\right)^{2}+p\left(\left(u^{\tau}\right)^{2}-1\right) d V_{\bar{g}}
$$

Because $u$ is a unit timelike vector, $1=-u^{\mu} u_{\mu}=\left(u^{\tau}\right)^{2}-\bar{g}(u, u)$, so if we define:

$$
\mathcal{E}_{0}(t)=\int_{\mathcal{D}_{t}} \epsilon\left(u^{\tau}\right)^{2}+p \bar{g}(u, u) d V_{\bar{g}}
$$

then (1.22) and (1.23) give:

$$
\mathcal{E}_{0}(t)=\mathcal{E}_{0}(0)+\int_{\mathcal{D}} T^{\mu \nu}\left(\nabla_{\mu} \tau_{\nu}\right) d V_{g} .
$$

We can write $T^{\mu \nu} \nabla_{\mu} \tau_{\nu}=\frac{1}{2} T^{\mu \nu}\left(\nabla_{\mu} \tau_{\nu}+\nabla_{\nu} \tau_{\mu}\right)=\frac{1}{2} T^{\mu \nu} \mathcal{L}_{\tau} g_{\mu \nu}$, where $\mathcal{L}$ denotes the Lie derivative. In the special case that $\tau$ is a Killing field (IE that $(M, g)$ is stationary), the second term in (1.26) vanishes and the energy (1.25) is conserved.

We would like a higher-order version of the energy (1.25). Following [6] and [26], the energies that we construct for the equations (1.16) have interior terms and a boundary term:

$$
\begin{aligned}
\mathcal{E}^{k, \ell}(t)=\frac{1}{2} \int_{\mathcal{D}_{t}}\left(\left(\tau_{\mu} \tau_{\nu}+\bar{g}_{\mu \nu}\right)\right. & \left.Q\left(\bar{\nabla}^{k} \nabla_{u}^{\ell} V^{\mu}, \bar{\nabla}^{k} \nabla_{u}^{\ell} V^{\nu}\right)\right) \\
+ & \frac{\sqrt{\sigma}}{4} \int_{\mathcal{D}_{t}} Q\left(\bar{\nabla}^{k} \nabla_{u}^{\ell} \sigma, \bar{\nabla}^{k} \nabla_{u}^{\ell} \sigma\right)\left(-V^{\tau}\right) e^{\prime}(\sigma) d V_{\bar{g}} \\
& \quad+\frac{1}{4} \int_{\partial \mathcal{D}_{t}} Q\left(\bar{\nabla}^{k} \nabla_{u}^{\ell} \sigma, \bar{\nabla}^{k} \nabla_{u}^{\ell} \sigma\right) \frac{\left(-V^{\tau}\right)}{\left|\nabla_{N} \sigma\right|} d V_{\gamma},
\end{aligned}
$$

Here, $\bar{u}$ is the spatial part of $u$ and $|\bar{u}|=\bar{g}(\bar{u}, \bar{u})$. See section 2 for notation. Recall that $u^{\tau}<0$ so the $\mathcal{E}^{k, \ell}$ are non-negative. Here, $\bar{\nabla}$ is the covariant derivative on $\mathcal{D}_{t}$, and $Q$ is a positive definite quadratic form which is the inner product of the tangential components on the boundary and increases to the full norm in the interior, which we define as follows. We let $\widetilde{\mathcal{N}}$ denote the outer unit conormal to $\partial \mathcal{D}_{t}$, and we let $\mathcal{N}$ denote an extension of $\widetilde{\mathcal{N}}$ to a neighborhood of the boundary. Writing $d=d(x)=\operatorname{dist}_{\bar{g}}\left(x, \partial \mathcal{D}_{t}\right)$ for the geodesic distance to the boundary, we let $\chi=\chi(d)$ denote a smooth function which is zero in the interior of $\mathcal{D}_{t}$ and one near $\partial \mathcal{D}_{t}$. We then define the projection:

$$
\prod_{i}^{j}=\delta_{i}^{j}-\chi(d) \mathcal{N}_{i} \mathcal{N}^{j}
$$


where $\mathcal{N}^{j}=\bar{g}^{j \ell} \mathcal{N}_{\ell}$ and Latin indices $i, j, k, \ldots$ run over $1,2,3$. For $x \in \partial \mathcal{D}_{t}$, this is the projection from $T_{x}\left(\mathcal{D}_{t}\right)$ onto $T_{x}\left(\partial \mathcal{D}_{t}\right)$, and in the interior it is the identity map. We will also write:

$$
\gamma^{i j}=\bar{g}^{i k} \prod_{k}^{j}
$$

for the metric on $\partial \mathcal{D}_{t}$ extended to a neighborhood of the boundary. If $\alpha, \beta$ are $(1, r)$ tensors on $\mathcal{D}$ then we define:

$$
Q\left(\alpha^{\mu}, \beta^{\nu}\right)=\gamma^{i_{1} j_{1}} \cdots \gamma^{i_{r} j_{r}} \alpha_{i_{1} \cdots i_{r}}^{\mu} \beta_{j_{1} \cdots j_{r}}^{\nu}
$$

For each $\mu, \nu, Q\left(\alpha^{\mu}, \beta^{\nu}\right)$ is the inner product of the spatial components of $\alpha$ and $\beta$ which are tangent to $\partial \mathcal{D}_{t}$, and in the interior of $\mathcal{D}_{t}$ it is the inner product of spatial components of $\alpha$ and $\beta$.

To control the curl we define:

$$
\mathcal{K}^{r}(t)=\int_{\mathcal{D}_{t}}\left|\operatorname{curl} \bar{\nabla}^{r-1} V\right|^{2} d V_{\bar{g}} .
$$

To control the enthalpy $\sigma$, we use the fact that $\sigma$ satisfies the following wave equation:

$$
-\nabla_{V}^{2} e(\sigma)+\frac{1}{2} \nabla_{\mu} \nabla^{\mu} \sigma=-\left(\nabla_{\mu} V^{\nu}\right)\left(\nabla_{\nu} V^{\mu}\right),
$$

which follows by taking the divergence of (1.16) and subtracting it from $\nabla_{V}$ applied to (1.17). Using (1.32), we are able to prove energy estimates for the quantities:

$$
\begin{aligned}
\mathcal{W}^{r}(t)=\frac{1}{2} \int_{\mathcal{D}_{t}}\left(\left(\nabla_{u}^{r+1} \sigma\right)^{2}+\Pi^{\mu \nu}\left(\nabla_{\mu} \nabla_{u}^{r} \sigma\right)\left(\nabla_{\nu} \nabla_{u}^{r} \sigma\right) \frac{1}{|\bar{u}|-u^{\tau}}\right) d V_{\bar{g}}+ & \\
& \frac{1}{2} \int_{\mathcal{D}_{t}}\left(\nabla_{u}^{r+1} \sigma\right)^{2}\left(\eta^{-2}-1\right)\left(-u^{\tau}\right) d V_{\bar{g}} .
\end{aligned}
$$

Recall that $\Pi$ is the projection onto the local simultaneuous space of the fluid, defined in (1.3). Here, $\eta$ is the sound speed, $\eta(\epsilon) \equiv \sqrt{p^{\prime}(\epsilon)}$. Since the speed of light is one in these units, we require $\eta \leq 1$, which makes the last term non-negative. We remark that for an equation of state with $\eta=1$, the operator on the right-hand side of (1.32) reduces to the wave operator in the metric $g$ and (1.33) reduces to the usual energy for the wave equation on $(M, g)$, ignoring the factor of $\left(|\bar{u}|-u^{\tau}\right)^{-1}$.

The energies we consider are then:

$$
\mathcal{E}^{r}(t)=\sum_{k+\ell=r}\left(\mathcal{E}^{k, \ell}(t)+\mathcal{K}^{k, \ell}\right)+\mathcal{W}^{r}(t) .
$$

In order to close our energy estimates, we will need to assume that the spatial part of the fluid velocity is bounded. We write:

$$
\lambda=\frac{|\bar{u}|}{\left|u^{\tau}\right|} .
$$

Note that $\lambda<1$ because $u$ is a unit timelike vector field. We assume that:

$$
\lambda \leq \lambda^{*},
$$

where $\lambda^{*}=\lambda^{*}(r)$ is a sufficiently small constant. See the discussion after equation (2.37).

We also need some a priori assumptions:

$$
\begin{aligned}
& |\nabla u|+|\nabla \sigma| \leq M_{1}, \text { in } \mathcal{D}_{t}, \\
& \left|\nabla^{2} \sigma\right| \leq M_{2} \text { on } \partial \mathcal{D}_{t} .
\end{aligned}
$$

In addition, we require that the equation of state $p=p(\epsilon)$ is reasonably well-behaved; see section 2.4 for the specific assumptions.

Our main theorem is then:

Theorem 1. Suppose that the above assumptions hold. For $r \geq 5$ there is a $T^{*}$ so that for $0 \leq t \leq T^{*}$ :

$$
\mathcal{E}^{r}(t) \leq 2 \mathcal{E}^{r}(0) .
$$


See theorem 2 for a precise version of this statement.

The quantities (1.27) do not directly control all space derivatives of $V$ and $\sigma$. To get back control over all derivatives of $V$, we use elliptic estimates as in [6], which require that we control $\overline{\operatorname{div}} V$ and $\overline{\operatorname{curl}} V$, the divergence and curl of $V$ taken over only spatial components; see section 2.1. To control $\overline{\operatorname{div}} V$, we will use equation (1.17) and to control $\overline{\operatorname{curl}} V$, we will use equation (1.16). In each case, we will need to be able to control quantities involving $\nabla_{\tau} V$, and we control this if we control $1 / u^{\tau}$ times $\nabla_{u} V$ and $\bar{u}^{\mu} \bar{\nabla}_{\mu} V$. We can then use the equation (1.16) and the fact that $\lambda$ is small to handle these terms. We will also use these elliptic estimates to control derivatives of $\sigma$.

An important aspect of these elliptic estimates, which are simple modifications of the estimates in [6] to the case of a non-Euclidean background metric, is that they only depend on the regularity of the second fundamental form $\theta$ of the boundary and do not rely on "straightening the boundary" is in the usual proof of the elliptic estimates in Sobolev spaces.

We therefore need to get estimates for $\theta$. For this we use another key idea from [6], which is that $q=0$ on $\partial \mathcal{D}_{t}$, then $\not 1 / \bar{\nabla}^{r} q$ can be controlled in terms of $\bar{\nabla}^{r-1} q$ on the boundary, provided that the second fundamental form $\theta$ is bounded. The basic idea is that if $q=0$ on $\partial \mathcal{D}_{t}, \not ा \bar{\nabla} q=0$ on $\partial \mathcal{D}_{t}$, and so:

$$
0=\left(\not I_{i}^{k} \bar{\nabla}_{k}\right)\left(\not \Pi_{j}^{\ell} \bar{\nabla}_{\ell}\right) q=\left(\not 1 \bar{\nabla}^{2}\right)_{i j} q+\Pi_{i}^{k}\left(\bar{\nabla}_{k} \not I_{j}^{\ell}\right) \bar{\nabla}_{\ell} q \text {. }
$$

Because $q=0$ on $\partial \mathcal{D}_{t}$, the second term here is:

$$
\Pi_{i}^{k} \bar{\nabla}_{k}\left(\mathcal{N}_{j} \mathcal{N}^{\ell}\right) \mathcal{N}_{\ell} \bar{\nabla}_{\mathcal{N} q}=\left(\Pi_{i}^{k} \bar{\nabla}_{k} \mathcal{N}_{j}\right) \bar{\nabla}_{N} q
$$

so we have:

$$
\left(\not \bar{\nabla} \bar{\nabla}^{2}\right)_{i j} q=\theta_{i j} \bar{\nabla}_{\mathcal{N}} q
$$

In particular, $\not \mid \bar{\nabla}^{2} q$ is lower order. Further, if $\left|\bar{\nabla}_{\mathcal{N}} q\right|>0$ and we have a bound for $\not I \bar{\nabla}^{2} q$, this gives a bound for $\theta$.

There is a higher-order version of this identity, which says that if $q=0$ on $\partial \mathcal{D}_{t}$ then:

$$
\not ा \bar{\nabla}^{r} q=\left(\nabla^{r-2} \theta\right) \bar{\nabla}_{\mathcal{N}} q+O\left(\bar{\nabla}^{r-1} q, \bar{\nabla}^{r-3} \theta\right) .
$$

See (4.22); a more precise version of this statement can be found in Proposition 4.3 in [6]. Using (1.43), we see that if the sign condition (1.12) holds, the boundary term in (1.27) controls $\left\|\bar{\nabla}^{r-2} \theta\right\|_{L^{2}\left(\partial \mathcal{D}_{t}\right)}$. Using the above ideas, in section 8 , we prove:

$$
\|V\|_{H^{r}\left(\mathcal{D}_{t}\right)}+\|\nabla \sigma\|_{H^{r}\left(\mathcal{D}_{t}\right)}+\|\theta\|_{H^{r-2}\left(\mathcal{D}_{t}\right)} \leq C \mathcal{E}^{r}(t) .
$$

See Lemma 8 and Proposition 6

\section{Definitions And ASSUmptions}

Throughout, we will use the convention that Greek letters $\mu, \nu, \ldots$ take values $0,1,2,3$ and Latin letters $i, j, \ldots$ take values $1,2,3$. We sum over any repeated upper and lower indices, and we will raise and lower indices with the metric $g$. We will write the components of $g$ as $g_{\mu \nu}$ and the components of the inverse metric as $g^{\mu \nu}$.

2.1. Spatial derivatives. We assume that $(M, g)$ admits a time function $\mathfrak{t}: M \rightarrow \mathbb{R}$ so that if $M_{t}=\{\mathfrak{t}=t\}$, then the $M_{t}$ are Cauchy surfaces and they foliate $M$. We then define the future directed timelike unit (co)normal to the time slice $M_{t}$ :

$$
\tau_{\mu}=\frac{1}{-\sqrt{g(\nabla \mathfrak{t}, \nabla \mathfrak{t})}} \nabla_{\mu} \mathfrak{t} \quad \tau^{\mu}=g^{\mu \nu} \tau_{\nu}
$$

as well as the projection from $T(M)$ to $T\left(M_{t}\right)$ :

$$
\bar{\Pi}_{\nu}^{\mu}=\delta_{\mu}^{\nu}+\tau^{\mu} \tau_{\nu}
$$

When $X$ is a four-vector we will write $\bar{X}^{\mu}=\Pi_{\nu}^{\mu} X^{\nu}$ and $X^{\tau}=\tau^{\mu} X_{\mu}$. If $\left(x^{1}, x^{2}, x^{3}\right)$ are local coordinates on $M_{t}$ and we write $t=x^{0}$, then $X=X^{\mu} \partial_{x^{\mu}}$ where:

$$
\bar{X}^{0}=0, \quad \bar{X}^{i}=X^{i}, i=1,2,3,
$$

and $X^{\tau}= \pm X^{0}$, with the minus sign when $X$ is future directed and the plus sign when $X$ is past directed. 
We let $\bar{g}$ denote the induced Riemannian metric on $M_{t}$ extended to be zero on the orthogonal complement of $T\left(M_{t}\right)$ in $T(M)$ :

$$
\bar{g}_{\mu \nu}=g_{\mu \nu}+\tau_{\mu} \tau_{\nu}, \quad \bar{g}^{\mu \nu}=g^{\mu \nu}+\tau^{\mu} \tau^{\nu}
$$

We will also write:

$$
\underline{g}_{\mu \nu}=\bar{g}_{\mu \nu}+\tau_{\mu} \tau_{\nu}, \quad \underline{g}^{\mu \nu}=\bar{g}^{\mu \nu}+\tau^{\mu} \tau^{\nu},
$$

and:

$$
|X|^{2}=\underline{g}_{\mu \nu} X^{\mu} X^{\nu}=\left(X^{\tau}\right)^{2}+\bar{g}^{\mu \nu} X_{\mu} X_{\nu}
$$

More generally, if $\beta$ is a $(0, r)$ tensor we write:

$$
|\beta|^{2}=\underline{g}^{\mu_{1} \nu_{1}} \cdots \underline{g}^{\mu_{r} \nu_{r}} \beta_{\mu_{1} \cdots \mu_{r}} \beta_{\nu_{1} \cdots \nu_{r}} .
$$

The spatial derivatives of a four-vector $X$ are:

$$
\bar{\nabla}_{\mu} X^{\nu}=\bar{\Pi}_{\mu}^{\mu^{\prime}} \bar{\Pi}_{\nu^{\prime}}^{\nu} \nabla_{\mu^{\prime}} X^{\nu^{\prime}}
$$

Writing $X^{\nu}=-\tau^{\nu} X^{\tau}+\bar{X}^{\nu}$, we see that:

$$
\bar{\nabla}_{\mu} X^{\nu}=-\left(\bar{\nabla}_{\mu} \tau^{\nu}\right) X^{\tau}+\bar{\nabla}_{\mu} \bar{X}^{\nu} .
$$

In particular if $X$ is tangent to $M_{t}$, this agrees with the intrinsic covariant derivative determined by $\bar{g}$.

The divergence of a four-vector $X$ is defined as:

$$
\operatorname{div} X=\operatorname{tr}_{g}(\nabla X)=\nabla_{\mu} X^{\mu}=\frac{1}{\sqrt{|\operatorname{det} g|}} \partial_{\mu}\left(\sqrt{|\operatorname{det} g|} X^{\mu}\right) .
$$

To define the curl, we let $\zeta_{X}$ denote the one-form associated to $X$ :

$$
\zeta_{X}=\left(\zeta_{X}\right)_{\mu} d x^{\mu}=g_{\mu \nu} X^{\nu} d x^{\mu}
$$

and then, if $d$ denotes the differential on $M$ :

$$
\operatorname{curl} X=d \zeta_{X}=\left(\partial_{\mu}\left(g_{\mu^{\prime} \nu} X^{\mu^{\prime}}\right)-\left(\partial_{\nu}\left(g_{\nu^{\prime} \mu} X^{\nu^{\prime}}\right)\right) d x^{\mu} d x^{\nu} .\right.
$$

When $X^{\mu}=\nabla^{\mu} \varphi$ for a function $\varphi: M \rightarrow \mathbb{R}, \operatorname{curl} X=0$.

There are analogous definitions of the divergence and curl intrinsic to $M_{t}$. If $W \in T\left(M_{t}\right)$ then we write:

$$
\overline{\operatorname{div}} W=\operatorname{tr}_{\bar{g}}(\bar{\nabla} W)=\bar{\nabla}_{\mu} W^{\mu}
$$

and we extend this to four-vectors $X$ by setting $\overline{\operatorname{div}} X=\overline{\operatorname{div}} \bar{X}$.

Writing:

$$
\bar{\zeta}_{W}=\left(\bar{\zeta}_{W}\right)_{i} d x^{i}=\bar{g}_{i j} W^{j} d x^{i}
$$

where $\left(x_{1}, x_{2}, x_{3}\right)$ is any coordinate system on $M_{t}$, we define:

$$
\overline{\operatorname{curl}} W=\bar{d} \zeta_{W}=\left(\left(\partial_{i}\left(\bar{g}_{j \ell} W^{\ell}\right)-\partial_{i}\left(\bar{g}_{i \ell} W^{\ell}\right)\right) d x^{i} d x^{j}\right.
$$

where $\bar{d}$ is the differential on $M_{t}$. This is a two-form on $M_{t}$ which we extend to a two-form on $M$ by setting:

$$
\overline{\operatorname{curl}} X(\tau, Y)=0 \text {, }
$$

for any vector $Y$. We will also let $\bar{\Delta}$ denote the Laplace-Beltrami operator determined by $\bar{g}$ :

$$
\bar{\Delta} q=\bar{\nabla}_{i} \bar{\nabla}^{i} q=\bar{g}^{i j} \bar{\nabla}_{i} \bar{\nabla}_{j} q=\overline{\operatorname{div}} \bar{\nabla} q .
$$

We let $R m$ denote the Riemann curvature tensor of $(M, g)$ :

$$
R m_{\mu \nu \alpha \beta} X^{\beta}=\nabla_{\mu} \nabla_{\nu} X_{\alpha}-\nabla_{\nu} \nabla_{\mu} X_{\alpha},
$$

If $\left(x^{0}, x^{1}, x^{2}, x^{3}\right)$ are local coordinates on $M$ then:

$$
R m_{\mu \nu \alpha}^{\beta}=\partial_{\mu} \Gamma_{\alpha \nu}^{\beta}-\partial_{\alpha} \Gamma_{\nu \mu}^{\beta}+\Gamma_{\nu \gamma}^{\beta} \Gamma_{\alpha \mu}^{\gamma}-\mathcal{G}_{\alpha \gamma}^{\beta} \mathcal{G}_{\nu \mu}^{\gamma},
$$

where the $\Gamma$ denote the Christoffel symbols of $g$ in this coordinate system.

It is convenient to assume a bound of the form:

$$
\sum_{s=1}^{N}\left|\nabla^{s} R m\right|+\left|\nabla^{s} \tau\right| \leq R
$$


for some constant $R$ and sufficiently large $N$. A simple consequence that we will use in section 4 is that:

$$
\nabla_{\pi(I)} \beta=\nabla_{I} \beta+\mathcal{R}(\beta)
$$

where $I=\left(i_{1}, \ldots, i_{s}\right)$ with $s \leq N, \pi$ is a permutation on $s$ letters and $\mathcal{R}$ satisfies the estimates:

$$
\|\mathcal{R}(\beta)\|_{L^{\infty}\left(M_{t}\right)} \leq R \sum_{j \leq s-2}\left\|\nabla^{j} \beta\right\|_{L^{\infty}\left(M_{t}\right)}, \quad\|\mathcal{R}(\beta)\|_{L^{2}\left(M_{t}\right)} \leq R \sum_{j \leq s-2}\left\|\nabla^{j} \beta\right\|_{L^{2}\left(M_{t}\right)} .
$$

It would not be difficult to prove our results with milder assumptions on $R m$ than (2.20), but this makes the statements and proofs of many of our theorems simpler.

2.2. The free boundary $\partial \mathcal{D}_{t}$. We set $\mathcal{D}_{t}=\mathcal{D} \cap M_{t}$, and we let $\mathcal{N}=\mathcal{N}_{i} d x^{i}$ denote the exterior unit conormal to $\partial \mathcal{D}_{t}$ in $M_{t}$. We will also write $N=N_{\mu} d x^{\mu}$ for the exterior unit conormal to $\Lambda$. We will use the metric $\bar{g}$ to raise and lower indices $i, j, k, \ldots$, so that $\mathcal{N}^{i}=\bar{g}^{i j} \mathcal{N}_{j}$. The projection from $T\left(\mathcal{D}_{t}\right)$ to $T\left(\partial \mathcal{D}_{t}\right)$ at the boundary is:

$$
\Pi_{i}^{j}=\delta_{j}^{i}-\mathcal{N}_{i} \mathcal{N}^{j}
$$

and the projection of an $(r, s)$ tensor $S$ to the boundary is:

$$
(\not \mid S)_{i_{1} \cdots i_{s}}^{j_{1} \cdots j_{r}}=\prod_{i_{1}}^{k_{1}} \cdots \prod_{i_{s}}^{k_{s}} \prod_{\ell_{1}}^{j_{1}} \cdots \prod_{\ell_{r}}^{j_{r}} S_{k_{1} \cdots k_{s}}^{\ell_{1} \cdots \ell_{r}}
$$

We let $\gamma$ denote the induced metric on $T\left(\partial \mathcal{D}_{t}\right)$, extended to be zero on the orthogonal complement in $T\left(\mathcal{D}_{t}\right)$ :

$$
\gamma_{i j}=\bar{g}_{i j}-\mathcal{N}_{i} \mathcal{N}_{j}, \quad \gamma^{i j}=\bar{g}^{i j}-\mathcal{N}^{i} \mathcal{N}^{j}
$$

We also write $d V_{\gamma}$ for the volume element on $\partial \mathcal{D}_{t}$.

We will use the following tangential spatial derivatives:

$$
\left.\nabla_{i} T^{j}=\not \Pi_{i}^{i^{\prime}} \not\right]_{j}^{j^{\prime}} \bar{\nabla}_{i^{\prime}} T^{j^{\prime}}
$$

When $T$ is tangent to $\partial \mathcal{D}_{t}$, this agrees with the intrinsic covariant derivative on $\partial \mathcal{D}_{t}$ determined by the metric $\gamma$.

The second fundamental form of $\partial \mathcal{D}_{t}$ is:

$$
\theta_{i j}=(\not / / \bar{\nabla} \mathcal{N})_{i j}
$$

We let $\iota_{0}$ denote the normal injectivity radius of $\mathcal{D}_{t}$. By definition this is the largest number $\iota_{0}$ so that the normal exponential map:

$$
\left(-\iota_{0}, \iota_{0}\right) \times \partial \mathcal{D}_{t} \rightarrow\left\{x \in M_{t} \mid \operatorname{dist}_{\bar{g}}\left(x, \partial \mathcal{D}_{t}\right)<\iota_{0}\right\}
$$

defined by:

$$
(\iota, \omega) \mapsto \omega+\iota \mathcal{N}(\omega),
$$

is injective. Here, dist $_{\bar{g}}$ denotes the geodesic distance with respect to $\bar{g}$. We will assume that the following assumption on the boundary holds:

$$
|\theta|+\frac{1}{\iota_{0}} \leq K \text { on } \partial \mathcal{D}_{t}
$$

2.3. Sobolev spaces. For a $(0, r)$-tensor $T$ on $\mathcal{D}$, recall that we are writing:

$$
|T|^{2}=\underline{g}^{\mu_{1} \nu_{1}} \cdots \underline{g}^{\mu_{r} \nu_{r}} T_{\mu_{1} \cdots \mu_{r}} T_{\nu_{1} \cdots \nu_{r}},
$$

where $\underline{g}$ is the Riemannian metric $\underline{g}_{\mu \nu}=\bar{g}_{\mu \nu}+\tau_{\mu} \tau_{\nu}$. We define:

$$
\|T\|_{L^{2}\left(\mathcal{D}_{t}\right)}^{2}=\int_{\mathcal{D}_{t}}|T|^{2} d V_{\bar{g}} \quad\|T\|_{H^{r}\left(\mathcal{D}_{t}\right)}^{2}=\sum_{\ell=0}^{r}\left\|\bar{\nabla}^{\ell} T\right\|_{L^{2}\left(\mathcal{D}_{t}\right)}^{2}
$$

as well as:

$$
\|T\|_{L^{2}\left(\partial \mathcal{D}_{t}\right)}^{2}=\int_{\partial \mathcal{D}_{t}}|T|^{2} d V_{\bar{g}}, \quad \quad\|T\|_{H^{r}\left(\partial \mathcal{D}_{t}\right)}^{2}=\sum_{\ell=0}^{r}\left\|\nabla^{\ell} T\right\|_{L^{2}\left(\partial \mathcal{D}_{t}\right)}
$$


2.4. Assumptions on the fluid variables. By definition, the fluid velocity $u$ is a future-directed timelike unit vector field:

$$
u^{\mu} u_{\mu}=-1, \quad u^{\tau}<0 .
$$

In particular note that $u^{\tau} \geq 1$ with equality only when $\bar{u}=0$. With $\lambda=\frac{|\bar{u}|}{\left|u^{\tau}\right|}$, we will assume that:

$$
\lambda \leq \lambda^{*}
$$

for some $\lambda^{*}$ which will be chosen sufficiently small. By (2.34), we always have $\lambda<1$. We now explain why we need this assumption. First, in (4.6) we will estimate $\bar{\nabla} V$ in terms of $\overline{\operatorname{div}} V, \overline{\operatorname{curl}} V$ and tangentially projected derivatives $\emptyset I \bar{\nabla} V$. The equation (1.17) gives us an equation for $\operatorname{div} V$, and by (7.11), we have:

$$
\overline{\operatorname{div}} V=\operatorname{div} V+\tau_{\mu} \nabla_{\tau} V^{\mu} .
$$

There is a similar formula relating $\overline{\operatorname{curl}} V$ and $\operatorname{curl} V$, and so to close the estimates we must control $\nabla_{\tau} V^{\mu}$. By definition:

$$
\nabla_{\tau}=\frac{1}{u^{\tau}}\left(\nabla_{u}-\bar{u}^{\mu} \nabla_{\mu}\right)
$$

This leads to an estimate of the form:

$$
|\bar{\nabla} V| \leq C\left(|\overline{\operatorname{div}} V|+|\overline{\operatorname{curl}} V|+\left|\nabla_{u} V\right|+\lambda|\bar{\nabla} V|+|\not| \bar{\nabla} V \mid\right) .
$$

We have equations for $\overline{\operatorname{div}} V, \overline{\operatorname{curl}} V$ and $\nabla_{u} V$, and the energy bounds the last term. If (2.35) holds, we are able to absorb the fourth term into the left-hand side. See Lemma 13.

We also use (2.35) to control $\sigma$ in section 5.3. By the wave equation (1.32) and using the fact that $\nabla_{V}$ preserves boundary conditions (by (1.19)), it is straightforward to prove estimates for $\nabla_{V}^{\ell} \sigma$. To control space derivatives, in (5.48) we re-write (1.32) as an elliptic equation of the form:

$$
\bar{\Delta} \sigma=e^{\prime}(\sigma) \nabla_{V}^{2} \sigma+\nabla_{\tau}^{2} \sigma+\ldots
$$

We can then use the elliptic estimates from [6] to control spatial derivatives. We will control $\nabla_{V}^{2} \sigma$ directly as above but we again need (2.35) to deal with the pure time derivatives of $\sigma$.

In [21], Oliynyk has also proved energy estimates for the system (1.16)-(1.19) using a different approach which avoids the assumption (2.35). There, the equations (1.16)-(1.17) and boundary conditions (1.18)-(1.19) are differentiated once in time and then reformulated as a system of wave equations with "acoustic" boundary conditions. In Lagrangian coordinates, one can use the "standard" elliptic estimate in fractional Sobolev spaces (see Theorem B.4 in [21, and Proposition B.3.1 in [9] for similar estimates) to get control over, in our notation, $\|V\|_{H^{r}\left(\mathcal{D}_{t}\right)}+\|\sigma\|_{H^{r+1}\left(\mathcal{D}_{t}\right)}+\|x\|_{H^{r+1 / 2}\left(\partial \mathcal{D}_{t}\right)}$, where $x$ denotes the Lagrangian coordinate map. This is different from our energy, where one instead controls the second fundamental form on the boundary in Sobolev spaces with an integer number of derivatives.

We will be considering a barotropic fluid, so that $p=p(\epsilon)$ for some smooth strictly increasing function $p$. We assume that:

$$
\left|\frac{d^{k}}{d \epsilon^{k}} p(\epsilon)\right| \leq L_{1}, k=1, \ldots, N,
$$

for sufficiently large $N$ to be determined later. The quantity $\eta(\epsilon) \equiv \sqrt{p^{\prime}(\epsilon)}$ is called the sound speed, and we will assume that there is a constant $L_{2}$ so that:

$$
0<L_{2} \leq \eta^{2} \leq 1 .
$$

Recall that we are working in units so that the speed of light is 1 , so this says the sound speed cannot exceed the speed of light.

Using (1.13) and the fact that $p$ is an invertible function of $\epsilon$, given any one of $p, \rho, \epsilon$, we can determine the other two. As mentioned in the introduction, we will work in terms of the enthalpy $\sigma$, which is defined by:

$$
\sqrt{\sigma}=\frac{\epsilon+p}{\rho} .
$$

We also need to assume a lower bound for $\sigma$ in $\mathcal{D}_{t}$ :

$$
0<L_{3} \leq \sigma .
$$

By (1.18), this holds automatically near $\partial \mathcal{D}_{t}$ with $L_{3}=\sigma_{0} / 2$, say. 
With $e(\sigma)=\log (\rho(\sigma) / \sqrt{\sigma})$, we assume:

$$
\left|\frac{d^{k}}{d \sigma^{k}} e(\sigma)\right| \leq L_{3}|e(\sigma)|, \quad \text { for } k=1, \ldots, N,
$$

for sufficiently large $N$. We write $L=L_{1}+1 / L_{2}+1 / L_{3}+L_{4}$.

An interesting equation of state satisfying the above conditions is the "two-phase model":

$$
p(\epsilon)= \begin{cases}\epsilon-\epsilon_{0}, & \epsilon>\epsilon_{0}, \\ 0, & \epsilon \leq \epsilon_{0}\end{cases}
$$

which was introduced in 2 as a model for the collapse of a spherically symmetric neutron star. (Note that Christodoulou uses $\rho, n$ to denote the energy density and mass density, respectively). This corresponds to sound speed $\eta=1$ in our notation, which is the largest physically realistic value. Such a model is known as a "stiff" or "incompressible" fluid; see [27, [25]. See also the recent work [28] where static solutions to the Einstein-Euler system (1.20) with this equation of state are studied.

We will also need to assume some bounds for derivatives of $u$ and $\sigma$ in $\mathcal{D}$ :

$$
\begin{array}{r}
|\nabla u|+|\nabla \sigma| \leq M_{1}, \text { in } \mathcal{D}_{t}, \\
\left|\nabla^{2} \sigma\right| \leq M_{2} \text { on } \partial \mathcal{D}_{t},
\end{array}
$$

and we will write $M=M_{1}+M_{2}$.

As mentioned in the introduction, we will assume that the following condition holds:

$$
-\bar{\nabla}_{\mathcal{N} p} \geq \delta>0 \text { on } \partial \mathcal{D}_{t}
$$

In the non-relativistic setting, the equations (1.1)-(1.11) are ill-posed unless (2.48) holds; see [1]. Note that by (1.19), writing $|\nabla p|_{g}^{2}=g(\nabla p, \nabla p)$, the condition (2.48) is equivalent to $\left|\nabla_{N} p\right| \geq C \delta>0$ for some constant $C=C(\lambda)$. By (1.13), $\frac{d}{d p} \sqrt{\sigma}=\frac{1}{\epsilon+p}$, so if (1.6)-(1.7) and (2.48) hold,

$$
-\nabla_{N} \sigma \geq \delta^{\prime} \equiv C \frac{\rho_{0}}{\sigma_{0}} \delta>0
$$

\section{LAGRANGIAN COORDINATES, THE EXTENSION OF THE NORMAL}

We now introduce a system of coordinates on $\mathcal{D}_{t}$, known as Lagrangian coordinates, which fixes the boundary $\partial \mathcal{D}_{t}$. Although all of our results are invariant under coordinate changes, it is convenient to prove some of the estimates in Lagrangian coordinates. In addition, we will need to use many of the results from 6] which are stated in Lagrangian coordinates. Let $\Omega$ be a domain in $\mathbb{R}^{3}$ and let $f: \mathcal{D}_{0} \rightarrow \Omega$ be a volume preserving diffeomorphism. If $V$ satisfies (1.11), we define the following vector field on $\mathcal{D}_{t}$ :

$$
W^{i}=\frac{1}{-V^{\tau}} \bar{V}^{i}
$$

Then the Lagrangian coordinates are a mapping $x:[0, T] \times \Omega \rightarrow \mathcal{D}$ defined by:

$$
\frac{d}{d t} x(t, y)=W(t, x(t, y))
$$

For each $t$, the map $x(t, \cdot)$ is a diffeomorphism from $\Omega$ to $\mathcal{D}_{t}$, and note that $x(t, \partial \Omega)=\partial \mathcal{D}_{t}$ by $(1.11)$.

We will use the letters $a, b, c \ldots$ to denote quantities expressed in the $y$-coordinates, and we will abuse notation and use $\bar{g}$ to also denote the induced Riemannian metric on $\Omega$ :

$$
\bar{g}_{a b}=\bar{g}_{i j} \frac{\partial y^{a}}{\partial x^{i}} \frac{\partial y^{b}}{\partial x^{j}}
$$

Similarly we write:

$$
\gamma_{a b}=\gamma_{i j} \frac{\partial y^{a}}{\partial x^{i}} \frac{\partial y^{b}}{\partial x^{j}}, \quad \quad \not / I_{b}^{a}=\bar{g}^{a c} \gamma_{b c}
$$


We now extend the projection $\prod$ to a neighborhood of the boundary. Let $d(y)=\operatorname{dist}_{\bar{g}}(y, \partial \Omega)$ denote the geodesic distance to the boundary, and let $\varphi$ be a function on $\Omega$ so that $\varphi=0$ on $\partial \Omega, \varphi<0$ in $\Omega$, and so that $|\bar{\nabla} \varphi|>0$ on $\partial \Omega$. Then the outer conormal and normal to $\partial \Omega$ are given by:

$$
\widetilde{\mathcal{N}}_{a}=\frac{\partial_{a} \varphi}{\bar{g}(\nabla \varphi, \nabla \varphi)}, \quad \widetilde{\mathcal{N}}^{a}=\bar{g}^{a b} \widetilde{\mathcal{N}}_{b}
$$

Let $\iota_{0}$ be as in (2.30) and let $\chi=\chi(d)$ denote a smooth positive function so that $\chi=1$ when $d \leq \iota / 4$ and so that $\chi=0$ when $d>\iota / 2$. We then extend the projection प్I (defined in (1.28)) to the interior by defining:

$$
\widetilde{\Pi}_{b}^{a}=\delta_{b}^{a}-\chi(d) \widetilde{N}^{a} \widetilde{N}_{b}
$$

Away from the boundary this is the identity map on $T(\Omega)$ and on $\partial \Omega$ this is the projection to $T(\partial \Omega)$. From now on we will write (3.6) as पI. We will also let $\gamma^{a b}$ denote the metric (3.4) extended to the interior as in (3.6).

The properties of the projection and its extension to the interior that we will use are:

Lemma 1. Let $h_{a b}=\nabla_{V} \bar{g}_{a b}$. On $[0, T] \times \partial \Omega$ :

$$
\nabla_{V} \gamma^{a b}=-\gamma^{a c} \gamma^{b d} h_{c d}
$$

and:

$$
\|\bar{\nabla} \gamma\|_{L^{\infty}(\Omega)} \leq C\left(\|\theta\|_{L^{\infty}(\partial \Omega)}+\frac{1}{\iota_{0}}\right), \quad \quad\left\|\nabla_{V} \gamma\right\|_{L^{\infty}(\Omega)} \leq C\|h\|_{L^{\infty}(\Omega)} .
$$

Proof. See Lemma 3.11 of [6].

We will write $\operatorname{Vol}(\Omega)$ for the volume of $\Omega$ with respect to the metric $\bar{g}$. The following lemma can be used to control $\operatorname{Vol}(\Omega)$ :

Lemma 2. With the above definitions, on $[0, T] \times \Omega$ :

$$
\frac{d}{d t} \operatorname{det} \bar{g}=\frac{\operatorname{det} \bar{g}}{V^{0}}\left(\overline{\operatorname{div}} V-V^{i} \partial_{i} \log V^{0}\right) .
$$

Proof. We abuse notation and write $V(t, y)=V(t, x(t, y))$ with $x$ from (3.2). By the well-known formula for the derivative of the determinant:

$$
\frac{d}{d t} \operatorname{det} \bar{g}(t, y)=\operatorname{det} \bar{g} \bar{g}^{a b}(t, y) \frac{d}{d t} \bar{g}_{a b}(t, y),
$$

and by (3.2), we have:

$$
\frac{d}{d t} \bar{g}_{a b}(t, y)=\left(\frac{d}{d t} \bar{g}_{i j}(t, y)\right) \frac{\partial x^{i}(t, y)}{\partial y^{a}} \frac{\partial x^{j}(t, y)}{\partial y^{b}}+2 \bar{g}_{i j}(t, y) \frac{\partial}{\partial y^{a}}\left(\frac{V^{i}(t, y)}{V^{0}(t, y)}\right) \frac{\partial x^{j}(t, y)}{\partial y^{b}} .
$$

The equation (3.10) then implies:

$$
\frac{d}{d t} \bar{g}(t, y)=\frac{\operatorname{det} \bar{g}(t, y)}{V^{0}(t, y)}\left(\partial_{i} V^{i}(t, y)-\frac{V^{i}(t, y)}{V^{0}(t, y)} \partial_{i} V^{0}(t, y)\right)
$$

\section{Elliptic Estimates}

We now fix a $(0,1)$ tensor $\alpha_{k}$ on $\mathcal{D}_{t}$ and let $\beta_{k}=\beta_{I k}=\bar{\nabla}_{I}^{r} \alpha_{k}$, where $\bar{\nabla}_{I}^{r}=\bar{\nabla}_{i_{1}} \cdots \bar{\nabla}_{i_{r}}$. We will also let $\beta_{I k}^{S}$ and $\beta_{I k}^{A}$ denote the symmetrization and anti-symmetrization of $\beta$ over the first $r$ indices respectively:

$$
\beta_{i_{1} \cdots i_{r} k}^{S}=\frac{1}{r !} \sum_{\pi \in S_{r}} \beta_{i_{p i(1)} \cdots i_{\pi(r)} k} \quad \beta_{i_{1} \cdots i_{r} k}^{A}=\frac{1}{r !} \sum_{\pi \in S_{r}}(-1)^{|\pi|} \beta_{i_{\pi(1)} \cdots i_{\pi(r)} k} .
$$

Here $S_{r}$ is the symmetric group on $r$ letters and $|\pi|$ is the order of the permutation $\pi$. Then $\beta=\beta^{S}+\beta^{A}$ and $\left|\beta^{S}\right|+\left|\beta^{A}\right| \leq 2|\beta|$. When $\bar{g}$ is flat then $\beta^{S}=\beta$ and $\beta^{A}=0$ but in general $\beta^{A}$ will involve derivatives of the Riemann curvature tensor.

We will rely on several elliptic estimates from []ㅡ, where it is assumed that $\left[\bar{\nabla}_{i}, \bar{\nabla}_{j}\right]=0$. To apply these estimates to the general case, we will write $\beta=\beta^{S}+\beta^{A}$ and many of the results from that paper can then 
be applied directly to $\beta^{S}$. Since we are assuming that we have bounds (2.20) for the Riemann tensor, $\beta^{A}$ will be lower order.

We will write:

$$
\overline{\operatorname{div}} \beta=\bar{g}^{i j} \bar{\nabla}_{i} \beta_{I j}, \quad \overline{\operatorname{curl}} \beta_{i j}=\nabla_{i} \beta_{I j}-\nabla_{j} \beta_{I i} .
$$

Note that by (2.20) we have:

$$
\overline{\operatorname{div}} \beta=\bar{\nabla}^{r} \overline{\operatorname{div}} \alpha+R_{1} \alpha, \quad \overline{\operatorname{curl}} \beta=\bar{\nabla}^{r} \overline{\operatorname{curl}} \alpha+R_{2} \alpha,
$$

where

$$
\left|R_{1} \alpha\right|+\left|R_{2} \alpha\right| \leq C(R) \sum_{k \leq r-1}\left|\bar{\nabla}^{k} \alpha\right|
$$

if $r \leq N$.

If $I=\left(i_{1}, \cdots, i_{r}\right), J=\left(j_{1}, \cdots j_{r}\right)$, we will write:

$$
\bar{g}^{I J}=\bar{g}^{i_{1} j_{1}} \cdots \bar{g}^{i_{r} j_{r}}, \quad \quad \gamma^{I J}=\gamma^{i_{1} j_{1}} \cdots \gamma^{i_{r} j_{r}} .
$$

The fundamental elliptic estimates are then:

Lemma 3. If $\beta$ is as above and the assumption (2.30) holds, then:

$$
\begin{gathered}
|\bar{\nabla} \beta|^{2} \leq C\left(\bar{g}^{i j} \gamma^{k \ell} \gamma^{I J} \bar{\nabla}_{k} \beta_{I i} \bar{\nabla}_{\ell} \beta_{J j}+|\overline{\operatorname{div}} \beta|^{2}+|\overline{\operatorname{curl}} \beta|^{2}+\left|\bar{\nabla} \beta^{A}\right|^{2}\right), \\
\int_{\Omega}|\bar{\nabla} \beta|^{2} d V_{\bar{g}} \leq C \int_{\Omega}\left(\mathcal{N}^{i} \mathcal{N}^{j} \bar{g}^{k \ell} \gamma^{I J} \bar{\nabla}_{k} \beta_{I i} \bar{\nabla}_{\ell} \beta_{J j}+|\overline{\operatorname{div}} \beta|^{2}+|\overline{\operatorname{curl}} \beta|^{2}+K|\beta|^{2}+\left|\bar{\nabla} \beta^{A}\right|^{2}\right) d V_{\bar{g}} .
\end{gathered}
$$

Proof. Write $\beta=\beta_{S}+\beta_{A}$ and apply the proof of lemma 5.5 from [6] to $\beta_{S}$.

Proposition 1. With $\beta$ as defined above, if (2.30) and (2.20) holds with $N \geq 1$ and $|\operatorname{Ric}(g)| \leq R^{\prime}$, then:

$$
\begin{gathered}
\|\beta\|_{L^{2}(\partial \Omega)}^{2} \leq C\left(\|\bar{\nabla} \beta\|_{L^{2}(\Omega)}+K\|\beta\|_{L^{2}(\Omega)}\right)\|\beta\|_{L^{2}(\Omega)}, \\
\|\beta\|_{L^{2}(\partial \Omega)}^{2} \leq C|| \not h \beta\left\|_{L^{2}(\partial \Omega)}^{2}+\right\| \beta^{A} \|_{L^{2}(\partial \Omega)}^{2} \\
\quad+C\left(\|\overline{\operatorname{div}} \beta\|_{L^{2}(\Omega)}+\|\overline{\operatorname{curl}} \beta\|_{L^{2}(\Omega)}+K\|\beta\|_{L^{2}(\Omega)}\right)\|\beta\|_{L^{2}(\Omega)},
\end{gathered}
$$

and

$$
\begin{aligned}
& \|\bar{\nabla} \beta\|_{L^{2}(\Omega)}^{2} \leq C|| \bar{\nabla} \beta\left\|_{L^{2}(\partial \Omega)}\right\| \beta \|_{L^{2}(\partial \Omega)} \\
& +C\left(\|\overline{\operatorname{div}} \beta\|_{L^{2}(\Omega)}+\|\overline{\operatorname{curl}} \beta\|_{L^{2}(\Omega)}\right)^{2}+\|\operatorname{Ric}(\bar{g})\|_{L^{\infty}(\Omega)}\|\beta\|_{L^{2}(\Omega)}^{2},
\end{aligned}
$$

$\|\bar{\nabla} \beta\|_{L^{2}(\Omega)}^{2} \leq C|| \not \backslash \bar{\nabla} \beta\left\|_{L^{2}(\partial \Omega)}\right\| \not h \mathcal{N} \cdot \beta \|_{L^{2}(\partial \Omega)}$

$$
+C\left(\|\overline{\operatorname{div}} \beta\|_{L^{2}(\Omega)}+\|\overline{\operatorname{curl}} \beta\|_{L^{2}(\Omega)}+(K+R)\|\beta\|_{L^{2}(\Omega)}^{2}\right)+\left\|\bar{\nabla} \beta^{A}\right\|_{L^{2}(\Omega)}^{2} .
$$

Here, Ric $(\bar{g})$ denotes the Ricci curvature of $(\Omega, \bar{g})$ and $\mathcal{N} \cdot \beta=\mathcal{N}^{\mu} \beta_{I \mu}$.

Proof. This is a straightforward modification of Lemma 5.6 from [6] and we just indicate the main points. First, (4.8) follows by Stokes' theorem:

$$
\int_{\partial \Omega} \underline{g}^{\mu \nu} \bar{g}^{I J} \beta_{I \mu} \beta_{J \nu} d V_{\gamma}=\int_{\Omega} \bar{\nabla}_{k}\left(\mathcal{N}^{k} \underline{g}^{\mu \nu} \bar{g}^{I J} \beta_{I \mu} \beta_{J \nu}\right) d V_{g} .
$$

The estimate (4.9) is proved by writing $\beta=\beta^{S}+\beta^{A}$ and applying the proof of (5.20) from [6] to $\beta^{S}$.

To prove (4.10) we use the "Hodge" identity:

$$
\begin{aligned}
\bar{\Delta} \beta_{k}=\bar{\nabla}^{i} \bar{\nabla}_{i} \beta_{k} & =\bar{\nabla}^{i} \bar{\nabla}_{k} \beta_{i}+\bar{\nabla}^{i}\left(\bar{\nabla}_{i} \beta_{k}-\bar{\nabla}_{k} \beta_{i}\right) \\
& =\bar{\nabla}_{k} \overline{\operatorname{div}} \beta+\bar{\nabla}^{i} \overline{\operatorname{curl}} \beta_{i k}+\operatorname{Ric}_{k \ell} \beta^{\ell},
\end{aligned}
$$

and then integrate by parts twice. 
Finally, to prove (4.11), we start with (4.7) and write $\mathcal{N}^{i} \mathcal{N}^{j} g^{k \ell}=\mathcal{N}^{i} \mathcal{N}^{k} g^{j \ell}+A^{i j k \ell}$ with $A^{i j k \ell}=$ $\mathcal{N}^{i} \mathcal{N}^{j} g^{k \ell}-\mathcal{N}^{i} \mathcal{N}^{k} g^{j \ell}$. We now modify the proof of (5.11) from [6]. Note that:

$$
\begin{aligned}
& A^{i j k \ell} \gamma^{I J} \bar{\nabla}_{k} \beta_{I i} \bar{\nabla}_{\ell} \beta_{J j} \\
& \quad=\bar{\nabla}_{k}\left(A^{i j k \ell} \gamma^{I J} \beta_{I i} \bar{\nabla}_{\ell} \beta_{J j}\right)-\bar{\nabla}_{k}\left(A^{i j k \ell}\right) \gamma^{I J} \beta_{I i} \bar{\nabla}_{\ell} \beta_{J j}-A^{i j k \ell} \gamma^{I J} \beta_{I i}\left(\overline{R m}_{k \ell j}^{m} \beta_{J m}\right),
\end{aligned}
$$

where $\overline{R m}$ is the Riemann curvature of $(\Omega, \bar{g})$, and in the last term we used that $A^{i j k \ell}=-A^{i k j \ell}$. Also note that on $\partial \Omega$ :

$$
\mathcal{N}_{k} A^{i j k \ell}=\mathcal{N}_{k}\left(\mathcal{N}^{i} \mathcal{N}^{j} g^{k \ell}-\mathcal{N}^{i} \mathcal{N}^{k} g^{j \ell}\right)=-\mathcal{N}^{i} \gamma^{j \ell}
$$

Integrating (4.15) over $\Omega$, using Stokes' theorem, this identity gives:

$$
\begin{aligned}
& \int_{\Omega} A^{i j k \ell} \gamma^{I J} \bar{\nabla}_{k} \beta_{I i} \bar{\nabla}_{\ell} \beta_{J j} d V_{\bar{g}} \\
& \leq\|\not \mid \bar{\nabla} \beta\|_{L^{2}(\partial \Omega)}\|\not \mathbf{Z}(\mathcal{N} \cdot \beta)\|_{L^{2}(\partial \Omega)}+\left(K\|\bar{\nabla} \beta\|_{L^{2}(\Omega)}+R\|\beta\|_{L^{2}(\Omega)}\right)\|\beta\|_{L^{2}(\Omega)} \\
& +C\left(\|\overline{\operatorname{div}} \beta\|_{L^{2}(\Omega)}^{2}+\|\overline{\operatorname{curl}} \beta\|_{L^{2}(\Omega)}^{2}+K\|\beta\|_{L^{2}(\Omega)}^{2}\right) .
\end{aligned}
$$

Combining this with (4.7) then implies (4.9).

The above estimates applied to $\beta=\bar{\nabla}^{\ell} q$ for a function $q$ give estimates that we will need to control solutions to the wave equation in the next section:

Proposition 2. Suppose that the assumptions (2.30) hold and (2.20) holds with $N \geq r$. If $q: \Omega \rightarrow \mathbb{R}$ and $r \geq 2$ :

$$
\begin{aligned}
\left\|\bar{\nabla}^{r} q\right\|_{L^{2}(\partial \Omega)}^{2}+\left\|\bar{\nabla}^{r} q\right\|_{L^{2}(\Omega)}^{2} \leq C & \left\|\not h \bar{\nabla}^{r} q\right\|_{L^{2}(\partial \Omega)}^{2} \\
& +C(K, R)\left(\|\bar{\Delta} q\|_{H^{r-1}(\Omega)}^{2}+\|\bar{\nabla} q\|_{L^{2}(\Omega)}^{2}+\|q\|_{L^{2}(\Omega)}^{2}+\|q\|_{L^{2}(\partial \Omega)}^{2}\right) .
\end{aligned}
$$

Furthermore, for any $\delta>0$ :

$$
\begin{aligned}
\left\|\bar{\nabla}^{r} q\right\|_{L^{2}(\Omega)}^{2}+\left\|\bar{\nabla}^{r-1} q\right\|_{L^{2}(\partial \Omega)}^{2} \leq C \delta\left\|\not h \bar{\nabla}^{r} q\right\|_{L^{2}(\partial \Omega)}^{2} & \\
& +C(K, R, 1 / \delta)\left(\|\bar{\Delta} q\|_{H^{r-2}(\Omega)}^{2}+\|\bar{\nabla} q\|_{L^{2}(\Omega)}^{2}+\|q\|_{L^{2}(\Omega)}\right) .
\end{aligned}
$$

Proof. This is a simple modification of the proof of Proposition 5.8 of [6], which relies on the previous proposition. The only ingredients needed are that, by (2.20),

$$
\left|\left(\bar{\nabla}^{r} q\right)^{A}\right| \leq R \sum_{k \leq r-2}\left|\bar{\nabla}^{k} q\right|
$$

and that by (4.3) we also have:

$$
\left\|\overline{\operatorname{div}} \bar{\nabla}^{r} q\right\|_{L^{2}(\Omega)}+\left\|\overline{\operatorname{curl}} \bar{\nabla}^{r} q\right\|\left\|_{L^{2}(\Omega)} \leq\right\| \bar{\nabla}^{r-1} \bar{\Delta} q\left\|_{L^{2}(\Omega)}+R\right\| q \|_{H^{r-2}(\Omega)}
$$

We will also need the following estimate for $\left\|\not / I \bar{\nabla}^{r} q\right\|_{L^{2}(\partial \Omega)}$ when $\not \nabla q=0$ on $\partial \Omega$, which is a simple modification of Proposition 5.9 from [6]:

Proposition 3. Suppose that $|\theta|+1 / \iota_{1} \leq K$. If $\not \nabla q=0$ on $\partial \Omega$, then for $m=0,1$ :

$$
\begin{aligned}
\left\|\not h \bar{\nabla}^{r} q\right\|_{L^{2}(\partial \Omega)} \leq C(K, R)\left(\left\|\left(\nabla^{r-2} \theta\right)\left(\bar{\nabla}_{\mathcal{N}} q\right)\right\|_{L^{2}(\partial \Omega)}\right. & +\sum_{k=1}^{r-1}\left\|\bar{\nabla}^{r-k} q\right\|_{L^{2}(\partial \Omega)} \\
& \left.+\left(\|\theta\|_{L^{\infty}(\partial \Omega)}+\|\theta\|_{H^{r-3}(\partial \Omega)}\right) \sum_{k=0}^{r-1}\left\|\bar{\nabla}^{k} q\right\|_{L^{2}(\partial \Omega)}\right)
\end{aligned}
$$

and if $r>3$, for any $\delta>0$ :

$$
\left\|\not h \bar{\nabla}^{r-1} q\right\|_{L^{2}(\partial \Omega)} \leq \delta\left\|\bar{\nabla}^{r-1} q\right\|_{L^{2}(\partial \Omega)}+C\left(1 / \delta, K,\|\theta\|_{H^{r-3}(\partial \Omega)}\right) \sum_{k=0}^{r-2}\left\|\bar{\nabla}^{k} q\right\|_{L^{2}(\partial \Omega)} .
$$




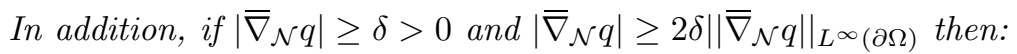

$$
\begin{aligned}
\left\|\nabla^{r-2} \theta\right\|_{L^{2}(\partial \Omega)} \leq C(1 / \delta, K, R)\left(\left\|\not \mid \overline{\nabla^{r}} q\right\|_{L^{2}(\partial \Omega)}\right. & +\sum_{k=1}^{r-1}\left\|\bar{\nabla}^{r-k} q\right\|_{L^{2}(\partial \Omega)} \\
& \left.+\left(\|\theta\|_{L^{\infty}(\partial \Omega)}+\|\theta\|_{H^{r-3}(\partial \Omega)}\right) \sum_{k \leq r-1}\left\|\bar{\nabla}^{k} q\right\|_{L^{2}(\partial \Omega)}\right),
\end{aligned}
$$

where the last lines in (4.24) and (4.22) are not present if $r \leq 4$.

Proof. Proposition 5.9 of [6] is stated for $q=0$ on $\partial \Omega$ but what is actually used is that $\not^{r} q=0$ (see inequality (4.5) there). As in the previous lemmas, we can apply the proof that proposition to $\left(\bar{\nabla}^{r} q\right)^{S}$, and bound $\left\|\left(\bar{\nabla}^{r} q\right)^{A}\right\|_{L^{2}(\partial \Omega)}$ by (2.21). The estimate (4.24) is similar.

The final result we need is a version of Proposition 5.8 from [6]:

Proposition 4. For any $0 \leq r \leq 4$, if $\bar{\nabla} q=0$ on $\partial \Omega$, then:

$$
\begin{aligned}
\left\|\bar{\nabla}^{r-1} q\right\|_{L^{2}(\partial \Omega)} \leq C\left(\|\left(\nabla^{r-3} \theta\right)\right. & \left.\left(\bar{\nabla}_{\mathcal{N}} q\right)\left\|_{L^{2}(\partial \Omega)}+\right\| \bar{\nabla}^{r-2} \bar{\Delta}_{q} \|_{L^{2}(\Omega)}\right) \\
& +C\left(K, \operatorname{Vol}(\Omega), R,\|\theta\|_{H^{r-4}(\partial \Omega)}\left(\left\|\bar{\nabla}_{N} q\right\|_{L^{\infty}(\partial \Omega)}+\|\bar{\Delta} q\|_{H^{r-3}(\Omega)}\right),\right.
\end{aligned}
$$

and if $r>3$ :

$$
\begin{aligned}
\left\|\bar{\nabla}^{r-1} q\right\|_{L^{2}(\partial \Omega)}+\|\bar{\nabla} q\|_{L^{\infty}(\partial \Omega)} \leq & C\left\|\bar{\nabla}^{r-2} \bar{\Delta} q\right\|_{L^{2}(\Omega)} \\
& +C\left(K, \operatorname{Vol}(\Omega), R,\|\theta\|_{L^{2}(\partial \Omega)}, \ldots,\left\|\nabla^{r-3} \theta\right\|_{L^{2}(\partial \Omega)}\right)\|\bar{\Delta} q\|_{H^{r-3}(\Omega)} .
\end{aligned}
$$

Proof. By (4.18) with $m=1$ and the estimate (4.22),

$$
\begin{aligned}
& \left\|\bar{\nabla}^{r-1} q\right\|_{L^{2}(\partial \Omega)} \leq C(K)\left(\| \bar{\nabla}^{r-3} \theta\right)\left(\bar{\nabla}_{\mathcal{N}} q\right)\left\|_{L^{2}(\partial \Omega)}+\right\| \bar{\Delta} q \|_{H^{r-2}(\Omega)} \\
& \left.+\left(\|\theta\|_{L^{\infty}(\partial \Omega)}+\|\theta\|_{H^{r-4}(\partial \Omega)}\right) \sum_{k=0}^{r-2}\left\|\bar{\nabla}^{k} q\right\|_{L^{2}(\partial \Omega)}^{2}+\|\bar{\nabla} q\|_{L^{2}(\Omega)}^{2}+\|q\|_{L^{2}(\Omega)}^{2}+\|q\|_{L^{2}(\partial \Omega)}^{2}\right) .
\end{aligned}
$$

Because $q=0$ on $\partial \Omega$, the last term vanishes and using (A.10), A.11) the third and second last terms are bounded by $C(\operatorname{Vol} \Omega)\|\bar{\Delta} q\|_{L^{2}(\Omega)}$. Repeating this argument and using (A.7) gives (4.25) and (4.26).

\section{Estimates For the acoustic Wave Equation}

Taking the divergence of (1.16) gives:

$$
\nabla_{\mu} \nabla_{V} V^{\mu}+\frac{1}{2} g^{\mu \nu} \nabla_{\mu} \nabla_{\nu} \sigma=\nabla_{V} \nabla_{\mu} V^{\mu}+\left(\nabla_{\mu} V^{\nu}\right)\left(\nabla_{\nu} V^{\mu}\right)+\frac{1}{2} g^{\mu \nu} \nabla_{\mu} \nabla_{\nu} \sigma,
$$

while applying $\nabla_{V}$ to (1.17) gives:

$$
\nabla_{V}^{2} e(\sigma)+\nabla_{V} \nabla_{\mu} V^{\mu}=0
$$

Therefore, $\sigma$ satisfies:

$$
\nabla_{V}^{2} e(\sigma)-\frac{1}{2} g^{\mu \nu} \nabla_{\mu} \nabla_{\nu} \sigma=\left(\nabla_{\mu} V^{\nu}\right)\left(\nabla_{\nu} V^{\mu}\right) .
$$

In this section, it is more convenient to work with $\nabla_{u}$ than of $\nabla_{V}$. Recalling that $V^{\mu}=\sqrt{\sigma} u^{\mu}$, the above is:

$$
\sigma \nabla_{u}^{2} e(\sigma)-\frac{1}{2} g^{\mu \nu} \nabla_{\mu} \nabla_{\nu} \sigma=\left(\nabla_{\mu} V^{\nu}\right)\left(\nabla_{\nu} V^{\mu}\right)-\left(\nabla_{u} e(\sigma)\right)\left(\nabla_{u} \sigma\right)
$$

By (1.13) and the definitions $e(\sigma)=\log (\rho(\sigma) / \sqrt{\sigma}), \eta=\sqrt{p^{\prime}(\epsilon)}$, we have:

$$
e^{\prime}(\sigma)=\frac{1}{2 \sigma}\left(\eta^{-2}-1\right)
$$


so the first term above is:

$$
\begin{aligned}
\sigma \nabla_{u}^{2} e(\sigma) & =\sigma e^{\prime}(\sigma) \nabla_{u}^{2} \sigma+\sigma\left(\nabla_{u} e^{\prime}(\sigma)\right) \nabla_{u} \sigma \\
& =\frac{1}{2}\left(\eta^{-2}-1\right) \nabla_{u}^{2} \sigma+\left(\nabla_{u} e^{\prime}(\sigma)\right)\left(\nabla_{u} \sigma\right)
\end{aligned}
$$

The equation (5.3) can be then written as:

$$
\left(\eta^{-2}-1\right) \nabla_{u}^{2} \sigma-\nabla_{\mu}\left(g^{\mu \nu} \nabla_{\nu} \sigma\right)=2\left(\nabla_{\mu} V^{\nu}\right)\left(\nabla_{\nu} V^{\mu}\right)-\left(\nabla_{u} e(\sigma)+\nabla_{u} e^{\prime}(\sigma)\right) \nabla_{u} \sigma
$$

Writing $g^{\mu \nu}=-u^{\mu} u^{\nu}+\Pi^{\mu \nu}$, we see that $\sigma$ satisfies the following Dirichlet problem:

$$
\begin{array}{ll}
\eta^{-2} \nabla_{u}^{2} \sigma-\nabla_{\mu}\left(\Pi^{\mu \nu} \nabla_{\nu} \sigma\right)=\mathcal{F}+\mathcal{G}(\sigma) & \text { in } \mathcal{D}_{t}, \\
\sigma=\sigma_{0} & \text { on } \partial \mathcal{D}_{t},
\end{array}
$$

where:

$$
\begin{aligned}
\mathcal{F} & =2\left(\nabla_{\mu} V^{\nu}\right)\left(\nabla_{\nu} V^{\mu}\right) \\
\mathcal{G}(\sigma) & =-\left(\nabla_{u} e(\sigma)+\nabla_{u} e^{\prime}(\sigma)\right) \nabla_{u} \sigma .
\end{aligned}
$$

The symmetric $(2,0)$-tensor $\eta^{-2} u^{\mu} u^{\nu}+\Pi^{\mu \nu}$ is known as the acoustical metric and (5.9) is known as the acoustic wave equation (see [17]). In this section we will derive estimates for the Dirichlet problem:

$$
\begin{aligned}
\eta^{-2} \nabla_{u}^{2} \psi-\nabla_{\mu}\left(\Pi^{\mu \nu} \nabla_{\nu} \psi\right) & =f, & & \text { in } \mathcal{D}_{t} \\
\psi & =C_{0}, & & \text { on } \partial \mathcal{D}_{t}, \\
\psi=\psi_{0}, \nabla_{u} \psi & =\psi_{1} & & \text { on } \mathcal{D}_{0} .
\end{aligned}
$$

where $C_{0}$ is a constant.

Let:

$$
\mathcal{E}_{W}(t)=\frac{1}{2} \int_{\mathcal{D}_{t}}\left(\left(\nabla_{u} \psi\right)^{2}+\Pi^{\mu \nu} \nabla_{\mu} \psi \nabla_{\nu} \psi\right) \frac{1}{|\bar{u}|-u^{\tau}} d V_{\bar{g}}+\frac{1}{2} \int_{\mathcal{D}_{t}}\left(\nabla_{u} \psi\right)^{2}\left(\eta^{-2}-1\right)\left(-u^{\tau}\right) d V_{\bar{g}} .
$$

Recall that we are writing:

$$
u^{\tau}=u^{\mu} \tau_{\mu}, \quad|\bar{u}|^{2}=\bar{g}(u, u) .
$$

Beacuse $u$ is timelike and future-directed, $u^{\tau}<0$ so the first term is non-negative. In addition, $\eta \leq 1$ so the second term is non-negative, though it degenerates if the sound speed is the same as the speed of light.

We also note that if:

$$
\widetilde{\mathcal{E}}(t)=\int_{\mathcal{D}_{t}}\left(\nabla_{\tau} \psi\right)^{2}+\bar{g}^{i j} \bar{\nabla}_{i} \psi \bar{\nabla}_{j} \psi d V_{\bar{g}}
$$

is the energy associated to the wave equation in the metric $g$, then:

$$
\widetilde{\mathcal{E}}(t) \leq C(\lambda, M) \mathcal{E}_{W}(t)
$$

which follows by writing $\nabla_{\tau}=\tau^{\mu} \nabla_{\mu}=-u^{\tau} \nabla_{u}+\Pi_{\nu}^{\mu} \tau^{\nu} \nabla_{\mu}$ and $\bar{\nabla}_{\mu}=-u_{\mu} \bar{\nabla}_{u}+\Pi_{\mu}^{\nu} \bar{\nabla}_{\nu}$ and the fact that $-\left(u^{\tau}+|\bar{u}|\right)$ is bounded above and below.

The basic energy estimate is:

Lemma 4. Suppose that the assumptions (2.40)-(2.46) hold. If $\psi$ satisfies (5.13) $-(5.14)$, then:

$$
\mathcal{E}_{W}(t) \leq C(M, \lambda, L, t)\left(\mathcal{E}_{W}(0)+\int_{\mathcal{D}}\left|f \nabla_{u} \psi\right| d V_{g}\right) .
$$

Proof. We multiply (5.13) by $\nabla_{u} \psi$ and integrate over $\mathcal{D}$ with respect to $d V_{g}$ :

$$
\begin{aligned}
& \int_{\mathcal{D}}\left(\eta^{-2} \nabla_{u}^{2} \psi \nabla_{u} \psi-\nabla_{\mu}\left(\Pi^{\mu \nu} \nabla_{\nu} \psi\right) \nabla_{u} \psi\right) d V_{g} \\
&=\frac{1}{2} \int_{\mathcal{D}} \eta^{-2} \nabla_{u}\left(\nabla_{u} \psi\right)^{2} d V_{g}+\int_{\mathcal{D}} \Pi^{\mu \nu}\left(\nabla_{\nu} \psi\right)\left(\nabla_{\mu} \nabla_{u} \psi\right) d V_{g} \\
& \quad-\int_{\Lambda} \Pi^{\mu \nu}\left(\nabla_{\nu} \psi\right)\left(\nabla_{u} \psi\right) N_{\mu} d V_{g}+\int_{\mathcal{D}_{t}} \mathcal{R}(t) d V_{\bar{g}}-\int_{\mathcal{D}_{0}} \mathcal{R}(0) d V_{\bar{g}}
\end{aligned}
$$


where:

$$
\mathcal{R}(t)=\left(\Pi^{\mu \nu} \tau_{\mu} \nabla_{\nu} \psi\right) \nabla_{u} \psi
$$

Beause $\psi=0$ on $\Lambda$ and $\nabla_{u}$ is tangential at the boundary, the integral over $\Lambda$ vanishes. The first term on the right-hand side is:

$$
\begin{aligned}
& \frac{1}{2} \int_{\mathcal{D}} \eta^{-2} \nabla_{u}\left(\nabla_{u} \psi\right)^{2} d V_{g} \\
& \quad=\frac{1}{2} \int_{\mathcal{D}_{t}} \eta^{-2}\left(-u^{\mu} \tau_{\mu}\right)\left(\nabla_{u} \psi\right)^{2} d V_{g}-\frac{1}{2} \int_{\mathcal{D}_{0}} \eta^{-2}\left(-u^{\mu} \tau_{\mu}\right)\left(\nabla_{u} \psi\right)^{2}-\frac{1}{2} \int_{\mathcal{D}} \nabla_{\mu}\left(u^{\mu} \eta^{-2}\right)\left(\nabla_{u} \psi\right)^{2} d V_{g} .
\end{aligned}
$$

Writing $\nabla_{\mu} \nabla_{u} \psi=\nabla_{u} \nabla_{\mu} \psi-\left(\nabla_{\mu} u^{\nu}\right) \nabla_{\nu} \psi$, the second term on the right-hand side of (5.21) is:

$$
\begin{aligned}
& \int_{\mathcal{D}} \Pi^{\mu \nu}\left(\nabla_{\nu} \psi\right)\left(\nabla_{u} \nabla_{\mu} \psi\right) d V_{g}-\int_{\mathcal{D}} \Pi^{\mu \nu}\left(\nabla_{\mu} u^{\alpha}\right)\left(\nabla_{\nu} \psi\right)\left(\nabla_{\alpha} \psi\right) d V_{g} \\
& \quad=\frac{1}{2} \int_{\mathcal{D}} \nabla_{u}\left(\Pi^{\mu \nu}\left(\nabla_{\nu} \psi\right)\left(\nabla_{\mu} \psi\right)\right) d V_{g}-\int_{\mathcal{D}}\left(\nabla_{u} \Pi^{\mu \nu}\right)\left(\nabla_{\mu} \psi\right)\left(\nabla_{\nu} \psi\right) d V_{g}-\int_{\mathcal{D}} \Pi^{\mu \nu}\left(\nabla_{\mu} u^{\alpha}\right)\left(\nabla_{\nu} \psi\right) \nabla_{\alpha} \psi d V_{g},
\end{aligned}
$$

and the first term here is:

$$
\begin{aligned}
& \frac{1}{2} \int_{\mathcal{D}} \nabla_{u}\left(\Pi^{\mu \nu}\left(\nabla_{\mu} \psi\right)\left(\nabla_{\nu} \psi\right)\right) d V_{g} \\
= & \frac{1}{2} \int_{\mathcal{D}_{t}}\left(-u^{\mu} \tau_{\mu}\right) \Pi^{\mu \nu}\left(\nabla_{\mu} \psi\right)\left(\nabla_{\nu} \psi\right) d V_{\bar{g}}-\frac{1}{2} \int_{\mathcal{D}_{t}}\left(-u^{\mu} \tau_{\mu}\right) \Pi^{\mu \nu}\left(\nabla_{\mu} \psi\right)\left(\nabla_{\nu} \psi\right) d V_{\bar{g}}-\int_{\mathcal{D}}\left(\nabla_{\mu^{\prime}} u^{\mu^{\prime}}\right) \Pi^{\mu \nu}\left(\nabla_{\mu} \psi\right)\left(\nabla_{\nu} \psi\right) d V_{g} .
\end{aligned}
$$

Writing

$$
M(t)=\frac{1}{2}\left(\eta^{-2}\left(\nabla_{u} \psi\right)^{2}+\Pi^{\mu \nu}\left(\nabla_{\mu} \psi\right)\left(\nabla_{\nu} \psi\right)\right)
$$

so far we have the identity:

$$
\begin{aligned}
& \int_{\mathcal{D}} f \nabla_{u} \psi d V_{g}=\int_{\mathcal{D}_{t}}\left(-u^{\tau}\right) M(t)+ \mathcal{R}(t) d V_{\bar{g}}-\int_{\mathcal{D}_{0}}\left(-u^{\tau}\right) M(0)-\mathcal{R}(0) d V_{\bar{g}} \\
&-\frac{1}{2} \int_{\mathcal{D}} \nabla_{\mu}\left(u^{\mu} \eta^{-2}\right)\left(\nabla_{u} \psi\right)^{2}+\left(\nabla_{\mu^{\prime}} u^{\mu^{\prime}}\right) \Pi^{\mu \nu}\left(\nabla_{\mu} \psi\right)\left(\nabla_{\nu} \psi\right) d V_{g} \\
& \quad-\int_{\mathcal{D}}\left(\nabla_{u} \Pi^{\mu \nu}\right)\left(\nabla_{\mu} \psi\right)\left(\nabla_{\nu} \psi\right) d V_{g}+\Pi^{\mu \nu}\left(\nabla_{\mu} u^{\alpha}\right)\left(\nabla_{\nu} \psi\right)\left(\nabla_{\alpha} \psi\right) d V_{g} .
\end{aligned}
$$

By the assumptions (2.40)-(2.44) on the equation of state and the bound (2.46), the terms on the second line are bounded by $C(M, L) \mathcal{E}_{W}(t)$, and by (5.19), the terms on the third line are bounded by $C(\lambda, M) \mathcal{E}_{W}(t)$.

To deal with the terms on $\mathcal{D}_{t}$, we first note that:

$$
\Pi^{\mu \nu} \tau_{\mu} \tau_{\nu}=g^{\mu \nu} \tau_{\mu} \tau_{\nu}+\left(u^{\tau}\right)^{2}=-1+\left(u^{\tau}\right)^{2}=\bar{g}(\bar{u}, \bar{u})=|\bar{u}|^{2}
$$

Therefore:

$$
\begin{aligned}
\left|\Pi^{\mu \nu} \nabla_{\nu} \psi \tau_{\nu}\right|\left|\nabla_{u} \psi\right| & \leq\left(\Pi^{\mu \nu} \tau_{\mu} \tau_{\nu}\right)^{1 / 2}\left(\Pi^{\mu \nu} \nabla_{\mu} \psi \nabla_{\nu} \psi\right)^{1 / 2}\left|\nabla_{u} \psi\right| \\
& \leq \frac{1}{2}|\bar{u}|\left(\left(\nabla_{u} \psi\right)^{2}+\Pi^{\mu \nu}\left(\nabla_{\mu} \psi\right)\left(\nabla_{\nu} \psi\right)\right)
\end{aligned}
$$

so, recalling that $u^{\tau}<0$ :

$$
\begin{aligned}
\left|\left(-u^{\tau}\right) M(t)+\mathcal{R}(t)\right| & \left.\geq \frac{1}{2} \mid\left(\left(-u^{\tau}\right) \eta^{-2}-|\bar{u}|\right)\left(\nabla_{u} \psi\right)^{2}+\left(\left(-u^{\tau}\right)-|\bar{u}|\right) \Pi^{\mu \nu} \nabla_{\mu} \psi \nabla_{\nu} \psi\right) \\
& =\frac{1}{2}\left|\left(-u^{\tau}-|\bar{u}|\right)\left(\left(\nabla_{u} \psi\right)^{2}+\Pi^{\mu \nu} \nabla_{\mu} \psi \nabla_{\nu} \psi\right)+\frac{1}{2}\left(\eta^{-2}-1\right)\left(-u^{\tau}\right)\left(\nabla_{u} \psi\right)^{2}\right| .
\end{aligned}
$$

Because $\eta \leq 1$, the last term is positive. Also note that we have:

$$
1=-u^{\mu} u_{\mu}=\left(u^{\tau}\right)^{2}-|\bar{u}|^{2}=\left(-u^{\tau}-|\bar{u}|\right)\left(-u^{\tau}+|\bar{u}|\right),
$$


so we have shown that:

$$
\mathcal{E}_{W}(t) \leq \mathcal{E}_{W}(0)+C(M, \lambda, L) \int_{0}^{t} \mathcal{E}_{W}(s) d s
$$

The estimate (5.20) then follows from Grönwall's integral inequality.

To control higher derivatives, we will use the fact that $\nabla_{u}$ is tangential at the boundary to control $\nabla_{u}^{\ell} \psi$, and then the elliptic estimates from section 4 to control space derivatives.

We define:

$$
\begin{aligned}
\mathcal{E}_{W}^{r}(t)=\frac{1}{2} \int_{\mathcal{D}_{t}}\left(\left(\nabla_{u}^{r+1} \psi\right)^{2}+\Pi^{\mu \nu}\left(\nabla_{\mu} \nabla_{u}^{r} \psi\right)\left(\nabla_{\nu} \nabla_{u}^{r} \psi\right)\right) & \frac{1}{|\bar{u}|-u^{\tau}} d V_{\bar{g}} \\
& +\frac{1}{2} \int_{\mathcal{D}_{t}}\left(\nabla_{u}^{r+1} \psi\right)^{2}\left(\eta^{-2}-1\right)\left(-u^{\tau}\right) d V_{\bar{g}} .
\end{aligned}
$$

We will also need some mixed norms in the interior:

$$
\|\psi(t)\|_{k, \ell}=\sum_{s \leq k, m \leq \ell}\left\|\bar{\nabla}^{s} \nabla_{u}^{m} \psi(t)\right\|_{L^{2}\left(\mathcal{D}_{t}\right)}, \quad \quad\|\psi(t)\|_{r}=\sum_{k+\ell \leq r}\|\psi(t)\|_{k, \ell}
$$

Note that $\|\psi(t)\|_{r, 0}+\|\psi(t)\|_{r-1,1} \leq C(M, \lambda, L) \mathcal{E}_{W}^{r-1}(t)$. We define:

$$
\begin{aligned}
f_{k, \ell} & =\bar{\nabla}^{k} \nabla_{u}^{\ell} f, \\
g_{k, \ell}^{1}(\psi) & =\bar{\nabla}^{k}\left(\bar{\Delta} \nabla_{u}^{\ell} \psi-\bar{\nabla}_{u}^{\ell} \bar{\Delta} \psi\right), \\
g_{k, \ell}^{2}(\psi) & =\bar{\nabla}^{k} \nabla_{u}^{\ell} \nabla_{\tau}^{2} \psi-\nabla_{\tau}^{2} \bar{\nabla}^{k} \bar{\nabla}_{u}^{\ell} \psi, \\
e_{k, \ell}(\psi) & =\eta^{-2} \bar{\nabla}^{k} \nabla_{u}^{\ell+2} \psi-\bar{\nabla}^{k} \nabla_{u}^{\ell}\left(\eta^{-2} \nabla_{u}^{2} \psi\right)
\end{aligned}
$$

as well as:

$$
\mathcal{R}_{r}=\sum_{k+\ell \leq r}\left\|f_{k, \ell}\right\|_{L^{2}(\Omega)}+\left\|g_{k, \ell}^{1}\right\|_{L^{2}(\Omega)}+\left\|g_{k, \ell}^{2}\right\|_{L^{2}(\Omega)}+\left\|e_{k, \ell}\right\|_{L^{2}(\Omega)}
$$

We will also write:

$$
\begin{aligned}
\Delta_{\Pi} & =\nabla_{\mu}\left(\Pi^{\mu \nu} \nabla_{\nu}\right) \\
\widetilde{g}_{r}(\psi) & =\Delta_{\Pi} \nabla_{u}^{r} \psi-\nabla_{u}^{\ell} \Delta_{\Pi} \psi
\end{aligned}
$$

Because $\nabla_{u}$ is tangential at the boundary, $\psi_{r}=\nabla_{u}^{r} \psi$ satisfies the wave equation:

$$
\begin{aligned}
\eta^{-2} \nabla_{u}^{2} \psi_{r}-\nabla_{\mu}\left(\Pi^{\mu \nu} \nabla_{\nu} \psi_{r}\right) & =f_{0, r}+\widetilde{g}_{0, r}(\psi)+e_{0, r}(\psi) & & \text { in } \Omega \\
\psi_{r} & =0 & & \text { on } \partial \Omega,
\end{aligned}
$$

and so by (5.20), we have:

$$
\mathcal{E}_{W}^{r}(t) \leq C\left(\mathcal{E}_{W}^{r}(0)+\int_{0}^{t}\left(\left\|f_{0, r}(s)\right\|_{L^{2}(\Omega)}+\left\|\widetilde{g}_{0, r}(s)\right\|_{L^{2}(\Omega)}+\left\|e_{0, r}(s)\right\|_{L^{2}(\Omega)}\right)\left\|\nabla_{u} \psi_{\ell}(s)\right\|_{L^{2}(\Omega)} d s\right),
$$

with $C=C(M, \lambda, L, t)$.

While it was convenient to use the decomposition $g^{\mu \nu}=-u^{\mu} u^{\nu}+\Pi^{\mu \nu}$ to control the material derivatives $\nabla_{u}$, to control the spatial derivatives it is simpler to use the decomposition $g^{\mu \nu}=-\tau^{\mu} \tau^{\nu}+\bar{g}^{\mu \nu}$. With $\bar{\Delta}$ the Laplace-Beltrami operator on $\mathcal{D}_{t}$ (defined in (2.17) ) we re-write (5.8) as an elliptic equation:

$$
\bar{\Delta} \sigma=\left(\eta^{-2}-1\right) \nabla_{u}^{2} \sigma+\nabla_{\mu}\left(\tau^{\mu} \tau^{\nu} \nabla_{\nu} \sigma\right)+\mathcal{F}+\mathcal{G}(\sigma)
$$

We will now prove estimates for the solution to the Dirichlet problem:

$$
\begin{array}{ll}
\bar{\Delta} \psi=\left(\eta^{-2}-1\right) \nabla_{u}^{2} \psi+\nabla_{\tau}^{2} \psi+f & \text { in } \Omega \\
\psi=C_{0} & \text { on } \partial \Omega,
\end{array}
$$

where $C_{0}$ is a constant. The term $\nabla_{u}^{2} \psi$ is lower order because it vanishes on the boundary. The term $\nabla_{\tau}^{2} \psi$ does not vanish on the boundary and is above top order, but we can use (2.35) to control it in terms of $\nabla_{u}^{2} \psi$ and a small parameter times $\bar{\nabla}^{2} \psi$.

Then the elliptic estimates from section 4 imply: 
Lemma 5. Under the above hypotheses, if $k+\ell=r$ with $r \geq 5$ :

$$
\begin{aligned}
&\|\psi(t)\|_{k, \ell} \leq C\left(\mathcal{E}_{W}^{r}(t)+\mathcal{R}_{r-1}(t)\right. \\
&\left.+\left(\|\theta(t)\|_{H^{r-2}\left(\partial \mathcal{D}_{t}\right)}+\|u(t)\|_{r-1}+P\left(M,\|u(t)\|_{r-2}\right)\right)\right)\|\psi(t)\|_{r-1}
\end{aligned}
$$

where $C=C\left(M, \lambda, L, K, R, \operatorname{Vol}\left(\mathcal{D}_{t}\right),\|\theta\|_{H^{2}\left(\partial \mathcal{D}_{t}\right)}\right)$.

Proof. The cases $k=0,1$ follow from (5.46). For $k \geq 2$ we use the elliptic estimate (4.19) and then(4) to control the term involving projected derivatives:

$$
\begin{aligned}
\left\|\bar{\nabla}^{k} \nabla_{u}^{\ell} \psi\right\|_{L^{2}\left(\mathcal{D}_{t}\right)} \leq C\left(\|\left(\nabla^{k-2} \theta\right)\right. & \left(\bar{\nabla}_{\mathcal{N}} \nabla_{u}^{\ell} \psi\right) \|_{L^{2}\left(\partial \mathcal{D}_{t}\right)} \\
& \left.+\left(\|\theta\|_{L^{\infty}\left(\partial \mathcal{D}_{t}\right)}+R\right)\left\|\bar{\nabla}^{k-2} \bar{\Delta} \nabla_{u}^{\ell} \psi\right\|_{L^{2}\left(\mathcal{D}_{t}\right)}+\widetilde{C}\left\|\bar{\Delta} \nabla_{u}^{\ell} \psi\right\|_{H^{k-3}\left(\mathcal{D}_{t}\right)}\right),
\end{aligned}
$$

where $\widetilde{C}=\widetilde{C}\left(K, R, \operatorname{Vol}\left(\mathcal{D}_{t}\right),\|\theta\|_{L^{\infty}\left(\partial \mathcal{D}_{t}\right)},\|\theta\|_{H^{k-3}\left(\partial \mathcal{D}_{t}\right)}\right)$.

When $k \leq r-2$, we bound the first term $\left\|\nabla^{k-2} \theta\right\|_{L^{\infty}\left(\partial \mathcal{D}_{t}\right)}\left\|\bar{\nabla}_{\mathcal{N}} \nabla_{u}^{\ell} \psi\right\|_{L^{2}\left(\partial \mathcal{D}_{t}\right)}$, and by Sobolev embedding (A.7) and the estimate (4.8) the result is bounded by the right-hand side of (5.50). For $k=r-1, r-2$, we bound this by $\left.\|\theta\|_{H^{r-2}\left(\partial \mathcal{D}_{t}\right)}|| \nabla \nabla_{u}^{\ell} \psi\left\|_{L^{\infty}\left(\partial \mathcal{D}_{t}\right)} \leq C\right\| \theta \|_{H^{r-2}\left(\partial \mathcal{D}_{t}\right)}\right) \mid \nabla \nabla_{u}^{\ell} \psi \|_{H^{2}\left(\partial \mathcal{D}_{t}\right)}$. Again by (4.8), this second factor is bounded by the right-hand side of (5.50) because $r \geq 5$.

To control $\left\|\bar{\nabla}^{k-2} \bar{\Delta} \nabla_{u}^{\ell} \psi\right\|_{L^{2}\left(\mathcal{D}_{t}\right)}$, we use (5.48) and (B.10):

$$
\begin{aligned}
\left\|\bar{\nabla}^{k-2} \bar{\Delta} \nabla_{u}^{\ell} \psi\right\|_{L^{2}\left(\mathcal{D}_{t}\right)} \leq C(L)\left\|\bar{\nabla}^{k-2} \nabla_{u}^{\ell+2} \psi\right\|_{L^{2}\left(\mathcal{D}_{t}\right)}+\lambda^{2}\left\|\bar{\nabla}^{k} \nabla_{u}^{\ell} \psi\right\|_{L^{2}\left(\mathcal{D}_{t}\right)} & +\mathcal{R}_{r-1}(t) \\
& +\|u\|_{r-1}\|\psi\|_{1}+P\left(M,\|u\|_{r-2}\right)\|\psi\|_{r-1} .
\end{aligned}
$$

For sufficiently small $\lambda$, returning to (5.51) we have:

$$
\begin{aligned}
\left\|\bar{\nabla}^{k} \nabla_{u}^{\ell} \psi\right\|_{L^{2}\left(\mathcal{D}_{t}\right)} \leq C\left(\left\|\bar{\nabla}^{k-2} \nabla_{u}^{\ell+2} \psi\right\|_{L^{2}\left(\mathcal{D}_{t}\right)}+\mathcal{R}_{r-1}(t)\right. & \\
& \left.+\left(\|\theta(t)\|_{H^{r-2}\left(\partial \mathcal{D}_{t}\right)}+\|u(t)\|_{r-1}+P\left(M,\|u(t)\|_{r-2}\right)\right)\right)\|\psi(t)\|_{r-1},
\end{aligned}
$$

with $C$ as in (5.50). Replacing $k$ with $k-2, \ell$ with $\ell+2$ and repeating this argument gives (5.50).

We finally return to the equation (5.9) satisfied by the enthalpy $\sigma$. We define:

$$
\begin{aligned}
\mathcal{W}^{r}(t)=\sum_{\ell \leq r} \int_{\mathcal{D}_{t}}\left(\left(\nabla_{u}^{r+1} \sigma(t)\right)^{2}+\Pi^{\mu \nu}\left(\nabla_{\mu} \nabla_{u}^{r} \sigma(t)\right)\left(\nabla_{\nu} \nabla_{u}^{r} \sigma(t)\right)\right) \frac{1}{|\bar{u}|-u^{\tau}} d V_{\bar{g}} & \\
& +\frac{1}{2} \int_{\mathcal{D}_{t}}\left(\nabla_{u}^{r+1} \sigma\right)^{2}\left(\eta^{-2}-1\right)\left(-u^{\tau}\right) d V_{\bar{g}} .
\end{aligned}
$$

Corollary 1. If $\sigma$ satifies (5.3) and the assumptions (2.20), (2.35), (2.40), (2.41), (2.46) and (2.47) hold, then there is a polynomial $P$ so that:

$$
\begin{aligned}
\mathcal{W}^{r}(t) \leq C\left(\mathcal{W}^{r}(0)+\int_{0}^{t}\left(\left\|\nabla^{r+1} \sigma(s)\right\|_{L^{2}\left(\mathcal{D}_{t}\right)}\right.\right. & \\
& \left.\left.+P\left(\|\sigma(s)\|_{r},\|V(s)\|_{r}, M, L, R\right)\right)\left\|\nabla_{u}^{r+1} \sigma(s)\right\|_{L^{2}\left(\mathcal{D}_{t}\right)} d s\right),
\end{aligned}
$$

and, for $k+\ell=r+1$ :

$$
\|\sigma\|_{k, \ell} \leq C\left(\mathcal{W}^{r}(t)+P\left(\|\sigma\|_{r},\|V\|_{r}, R\right)\right)
$$

where $C=C\left(t, M, \lambda, K,\|\theta\|_{H^{r-2}\left(\partial \mathcal{D}_{t}\right)}, \operatorname{Vol}\left(\mathcal{D}_{t}\right), L\right)$. 
Proof. We write:

$$
\begin{array}{r}
\mathcal{F}_{k, \ell}=\bar{\nabla}^{k} \nabla_{u}^{\ell} \mathcal{F}, \\
\mathcal{G}_{k, \ell}(\sigma)=\bar{\nabla}^{k} \nabla_{u}^{\ell} \mathcal{G}(\sigma),
\end{array}
$$

where $\mathcal{F}, \mathcal{G}$ are defined in (5.11)-(5.12)

By Lemma 15] in the appendix we have the following estimates when $k+\ell=r$ :

$$
\begin{array}{r}
\left\|\mathcal{F}_{k, \ell}\right\|_{L^{2}\left(\mathcal{D}_{t}\right)} \leq C(M, \lambda)\left(\left\|\nabla \bar{\nabla}^{k} \nabla_{u}^{\ell} V\right\|_{L^{2}\left(\mathcal{D}_{t}\right)}+P\left(\|V\|_{r},\|\sigma\|_{r}, R\right)\right), \\
\left\|g_{k, \ell}^{1}(\sigma)\right\|_{L^{2}\left(\mathcal{D}_{t}\right)}+\left\|g_{k, \ell}^{2}(\sigma)\right\|_{L^{2}\left(\mathcal{D}_{t}\right)}+\left\|\widetilde{g}_{r}(\sigma)\right\|_{L^{2}\left(\mathcal{D}_{t}\right)} \leq C(M, \lambda)\left(\left\|\nabla^{k+\ell+1} u\right\|_{L^{2}\left(\mathcal{D}_{t}\right)}+P\left(\|u\| \|_{r}\right)\|\sigma\|_{r}\right), \\
\left\|e_{k, \ell}(\sigma)\right\|_{L^{2}\left(\mathcal{D}_{t}\right)}+\left\|\mathcal{G}_{k, \ell}(\sigma)\right\|_{L^{2}\left(\mathcal{D}_{t}\right)} \leq C(M, \lambda, L)\left(\left\|\bar{\nabla}^{k} \nabla_{u}^{\ell} \sigma\right\|_{L^{2}\left(\mathcal{D}_{t}\right)}+P\left(\|u\|_{r-1},\|\sigma\|_{r-1}\right)\right),
\end{array}
$$

for some polynomials $P$.

Then (5.55) and (5.56) follow from (5.46) and (5.50).

\section{The ENERGY IDENTITY}

We let $\beta_{\mu}=\beta_{I \mu}$ and $\alpha_{\mu}=\alpha_{I \mu}$. As in previous sections, we will write $\bar{\beta}_{\mu}=\bar{\Pi}_{\mu}^{\nu} \beta^{\nu}$ as well as $\operatorname{div} \beta=$ $g^{\mu \nu} \nabla_{\mu} \beta_{I \nu}$. We will also write:

$$
\overline{\operatorname{div}} \alpha=\bar{g}^{\mu \nu} \bar{\nabla}_{\mu} \alpha_{I \nu}, \quad \overline{\operatorname{curl}} \alpha_{\mu \nu}=\bar{\nabla}_{\mu} \alpha_{I \nu}-\bar{\nabla}_{\nu} \alpha_{I \mu}
$$

We let $\not I$ be the projection to $\partial \mathcal{D}_{t}$ extended to the interior, defined in (3.6). If $\omega$ is a $(0, r)$ tensor we set:

$$
Q(\omega, \omega)=\bar{g}^{I J}\left(\prod_{I}^{K} \omega_{K}\right)\left(\Pi_{J}^{L} \omega_{L}\right)=\bar{g}^{i_{1} j_{1}} \cdots \bar{g}^{i_{r} j_{r}}\left(\prod_{i_{1}}^{k_{1}} \cdots \prod_{i_{r}}^{k_{r}} \omega_{k_{1} \cdots k_{r}}\right)\left(\Pi_{j_{1}}^{\ell} \cdots \prod_{j_{r}}^{\ell_{r}} \omega_{\ell_{1} \cdots \ell_{r}}\right)
$$

and then we define:

$$
\begin{aligned}
E(t)=\frac{1}{2} \int_{\mathcal{D}_{t}}\left(\left(\tau^{\mu} \tau^{\nu}+\bar{g}^{\mu \nu}\right) \gamma^{i j} Q\right. & \left.\left(\bar{\nabla}_{i} \beta_{\mu}, \bar{\nabla}_{j} \beta_{\nu}\right)\right) \frac{\sqrt{\sigma}}{|\bar{u}|-u^{\tau}} d V_{\bar{g}} \\
& +\frac{1}{2} \int_{\mathcal{D}_{t}} \gamma^{i j} Q\left(\bar{\nabla}_{i} \alpha, \bar{\nabla}_{j} \alpha\right)\left(-V^{\tau}\right) \kappa d V_{\bar{g}} \\
& \quad+\frac{1}{2} \int_{\partial \mathcal{D}_{t}} \gamma^{i j} Q\left(\bar{\nabla}_{i} \alpha, \bar{\nabla}_{j} \alpha\right)\left(-V^{\tau}\right) \nu d V_{\gamma},
\end{aligned}
$$

where $0<\nu, \kappa<\infty$. Suppose that the following bounds for $\nu, \kappa$ and $\partial \mathcal{D}_{t}$ hold:

$$
\begin{array}{ll}
\left|\frac{\nabla_{V} \kappa}{\kappa}\right| \leq C_{1}, & \text { in } \mathcal{D}_{t}, \\
\left|\frac{\nabla_{V} \nu}{\nu}\right| \leq C_{2}, & \text { on } \partial \mathcal{D}_{t} \\
|\theta|+\frac{1}{\iota_{0}} \leq K, & \text { on } \partial \mathcal{D}_{t} .
\end{array}
$$

Then we have the following energy estimate:

Proposition 5. If the assumptions (6.4) -(6.6) and (2.20) hold, then:

$$
\begin{aligned}
E(t)-E(0) & \leq \int_{0}^{t} \sqrt{E(s)}\left(\left\|\nabla_{V} \bar{\nabla} \beta+\nabla \bar{\nabla} \alpha\right\|_{L^{2}\left(\mathcal{D}_{s}\right)}+\left\|\operatorname{div} \bar{\nabla} \beta+\kappa \nabla_{V} \bar{\nabla} \alpha\right\|_{L^{2}\left(\mathcal{D}_{s}\right)}\right. \\
& \left.+\left\|\Pi\left(N^{\mu} \bar{\nabla} \beta_{\mu}-\nu \nabla_{V} \bar{\nabla} \alpha\right)\right\|_{L^{2}\left(\partial \mathcal{D}_{s}\right)}+\left\|\bar{\nabla} \alpha+V^{\mu} \bar{\nabla} \beta_{\mu}\right\|_{L^{2}\left(\mathcal{D}_{s}\right)}\right) \\
& +C(R)\left(\left(C_{1}+C_{2}+K\right) E(s)+\left(\|\overline{\operatorname{div}} \alpha\|_{L^{2}\left(\mathcal{D}_{s}\right)}^{2}+\|\overline{\operatorname{curl}} \alpha\|_{L^{2}\left(\mathcal{D}_{s}\right)}^{2}\right.\right. \\
& \left.+\|\overline{\operatorname{div}} \beta\|_{L^{2}\left(\mathcal{D}_{s}\right)}^{2}+\|\overline{\operatorname{curl}} \beta\|_{L^{2}\left(\mathcal{D}_{s}\right)}^{2}\right) d s .
\end{aligned}
$$


Remark. We will apply the above lemma with $\beta=\bar{\nabla}^{r-1} V, \alpha=\frac{1}{2} \bar{\nabla}^{r-1} \sigma, \kappa=2 e^{\prime}(\sigma)$ and $\nu=\frac{2}{\left|\nabla_{N} \sigma\right|}$. The first and second terms on the right-hand side then vanish to highest order by (1.16) and (1.17). The third term is a tangential projection पI of:

$$
N^{\mu} \bar{\nabla}^{r} V_{\mu}-\frac{1}{\left|\nabla_{N} \sigma\right|} \nabla_{V} \nabla^{r} \sigma=-\frac{1}{\left|\nabla_{N} \sigma\right|}\left(\nabla^{\mu} \sigma \nabla^{r} V_{\mu}+\nabla_{V} \nabla^{r} \sigma\right)
$$

To highest order, $\nabla^{\mu} \sigma \nabla^{r} V_{\mu}+\nabla_{V} \nabla^{r} \sigma=\nabla^{r} \nabla_{V} \sigma$, and because $\nabla_{V} \sigma=0, \not I \nabla^{r} \nabla_{V} \sigma$ is lower order. The fourth term will be lower order because $\sigma=-V^{\mu} V_{\mu}$.

Proof. We multiply $\nabla_{V} \bar{\nabla}_{i} \beta_{I \mu}+\nabla_{\mu} \bar{\nabla}_{i} \alpha_{I}$ by $\gamma^{i j} \gamma^{I J} g^{\mu \nu} \bar{\nabla}_{j} \beta_{J \nu}$ and integrate over $\mathcal{D}$ to get:

$$
\begin{aligned}
\int_{\mathcal{D}} \gamma^{i j} \gamma^{I J} g^{\mu \nu}\left(\left(\nabla_{V} \bar{\nabla}_{i} \beta_{I \mu}\right)\left(\bar{\nabla}_{j} \beta_{J \nu}\right)+\left(\nabla_{\mu} \bar{\nabla}_{i} \alpha_{I}\right)\left(\bar{\nabla}_{j} \beta_{J \nu}\right)\right) d V_{g} \\
\quad=\int_{\mathcal{D}} \gamma^{i j} \gamma^{I J} g^{\mu \nu}\left(\frac{1}{2} \nabla_{V}\left(\left(\bar{\nabla}_{i} \beta_{I \mu}\right)\left(\bar{\nabla}_{j} \beta_{J \nu}\right)\right)-\left(\bar{\nabla}_{i} \alpha_{I}\right)\left(\nabla_{\mu} \bar{\nabla}_{j} \beta_{J \nu}\right)\right) d V_{g} \\
\quad-\int_{\mathcal{D}_{t}} \gamma^{i j} \gamma^{I J}\left(\bar{\nabla}_{i} \alpha_{I}\right)\left(\bar{\nabla}_{j} \beta_{I \mu} \tau^{\mu}\right) d V_{\bar{g}}+\int_{\mathcal{D}_{0}} \gamma^{i j} \gamma^{I J}\left(\bar{\nabla}_{i} \alpha_{J}\right)\left(\bar{\nabla}_{j} \beta_{I \mu} \tau^{\mu}\right) d V_{\bar{g}} \\
\quad+\int_{\Lambda} \gamma^{i j} \gamma^{I J}\left(\bar{\nabla}_{i} \alpha_{I}\right)\left(\bar{\nabla}_{j} \beta_{J \mu}\right) N_{\mu} d V_{\gamma}-\int_{\mathcal{D}} \nabla_{\mu}\left(\gamma^{i j} \gamma^{I J}\right) g^{\mu \nu}\left(\bar{\nabla}_{i} \alpha_{I}\right)\left(\bar{\nabla}_{j} \beta_{J \nu}\right) d V_{g}
\end{aligned}
$$

The first term on the second line is:

$$
\begin{aligned}
\frac{1}{2} \int_{\mathcal{D}_{t}} \gamma^{i j} g^{\mu \nu} Q\left(\bar{\nabla}_{i} \beta_{\mu}, \bar{\nabla}_{j} \beta_{\nu}\right)\left(-V^{\tau}\right) d V_{\bar{g}}-\frac{1}{2} \int_{\mathcal{D}_{0}} \gamma^{i j} g^{\mu \nu} Q\left(\bar{\nabla}_{i} \beta_{\mu}, \bar{\nabla}_{j} \beta_{\nu}\right)\left(-V^{\tau}\right) d V_{\bar{g}} \\
\frac{1}{2} \int_{\Lambda} \gamma^{i j} g^{\mu \nu} Q\left(\bar{\nabla}_{i} \beta_{\mu}, \bar{\nabla}_{j} \beta_{\nu}\right) V^{\mu^{\prime}} N_{\mu^{\prime}}-\frac{1}{2} \int_{\mathcal{D}} \nabla_{\mu^{\prime}}\left(\gamma^{i j} \gamma^{I J} V^{\mu^{\prime}}\right) g^{\mu \nu}\left(\bar{\nabla}_{i} \beta_{I \mu}\right)\left(\bar{\nabla}_{j} \beta_{J \nu}\right),
\end{aligned}
$$

and the term on $\Lambda$ vanishes by the boundary condition (1.11).

The second term on the second line of (6.15) is:

$$
\begin{aligned}
\int_{\mathcal{D}} \gamma^{i j} \gamma^{I J}\left(\bar{\nabla}_{i} \alpha_{I}\right)\left(\nabla_{V} \bar{\nabla}_{j} \alpha_{J}\right) \kappa d V_{g}-\int_{\mathcal{D}} \gamma^{i j} \gamma^{I J}\left(\bar{\nabla}_{i} \alpha_{I}\right)\left(\operatorname{div} \bar{\nabla}_{j} \beta_{J}+\kappa \nabla_{V} \bar{\nabla}_{j} \alpha_{J}\right) d V_{g} \\
=\frac{1}{2} \int_{\mathcal{D}_{t}} \gamma^{i j} \gamma^{I J}\left(\bar{\nabla}_{i} \alpha_{I}\right)\left(\bar{\nabla}_{j} \alpha_{J}\right) \kappa\left(-V^{\tau}\right) d V_{\bar{g}}-\frac{1}{2} \int_{\mathcal{D}_{0}} \gamma^{i j} \gamma^{I J}\left(\bar{\nabla}_{i} \alpha_{I}\right)\left(\bar{\nabla}_{j} \alpha_{J}\right) \kappa\left(-V^{\tau}\right) d V_{\bar{g}} \\
\quad-\int_{\mathcal{D}} \nabla_{\mu}\left(\gamma^{i j} \gamma^{I J} \kappa V^{\mu}\right)\left(\bar{\nabla}_{i} \alpha_{I}\right)\left(\bar{\nabla}_{j} \alpha_{J}\right)-\gamma^{i j} \gamma^{I J}\left(\bar{\nabla}_{i} \alpha_{I}\right)\left(\operatorname{div} \bar{\nabla}_{j} \beta_{J}+\kappa \nabla_{V} \bar{\nabla}_{j} \alpha_{J}\right) d V_{g}
\end{aligned}
$$

The integral over $\Lambda$ from (6.15) is:

$$
\begin{aligned}
\int_{\Lambda} \gamma^{i j} Q\left(\bar{\nabla}_{i} \alpha, \bar{\nabla}_{j} \beta_{\mu}\right) N^{\mu} & d V_{\gamma} \\
& =\int_{\Lambda} \gamma^{i j} Q\left(\bar{\nabla}_{i} \alpha, \nabla_{V} \bar{\nabla}_{j} \alpha\right) \nu d V_{\gamma}+\int_{\Lambda} \gamma^{i j} Q\left(\bar{\nabla}_{i} \alpha,\left(\bar{\nabla}_{j} \beta_{\mu}\right) N^{\mu}-\nu \nabla_{V} \bar{\nabla} \alpha\right) d V_{\gamma} .
\end{aligned}
$$

By the boundary condition (1.11), $\nabla_{V}=V^{\mu} \nabla_{\mu}=V^{\mu} \not_{\mu}$, where $\not \nabla$ is the intrinsic covariant derivative on $\Lambda$. Since the boundary of $\Lambda$ is $\partial \mathcal{D}_{t} \cup \partial \mathcal{D}_{0}$, applying the divergence theorem on $\Lambda$ gives:

$$
\begin{aligned}
& \int_{\Lambda} \gamma^{i j} \gamma^{I J}\left(\bar{\nabla}_{i} \alpha_{I}\right)\left(\nabla_{V} \bar{\nabla}_{j} \alpha_{J}\right) \nu d V_{\gamma} \\
&=\frac{1}{2} \int_{\partial \mathcal{D}_{t}} \gamma^{i j} \gamma^{I J}\left(\bar{\nabla}_{i} \alpha_{I}\right)\left(\bar{\nabla}_{j} \alpha_{J}\right)\left(-V^{\mu} \tau_{\mu}\right) \nu d V_{\gamma}-\frac{1}{2} \int_{\partial \mathcal{D}_{0}} \gamma^{i j} \gamma^{I J}\left(\bar{\nabla}_{i} \alpha_{I}\right)\left(\bar{\nabla}_{j} \alpha_{J}\right)\left(-V^{\mu} \tau_{\mu}\right) \nu d V_{\gamma} \\
& \quad-\int_{\Lambda} \nabla_{\mu}\left(\gamma^{i j} \gamma^{I J} V^{\mu} \nu\right)\left(\bar{\nabla}_{i} \alpha_{I}\right)\left(\bar{\nabla}_{j} \alpha_{J}\right) d V_{\gamma} .
\end{aligned}
$$


If we write $\bar{\nabla}_{i} \alpha=-V^{\nu} \bar{\nabla}_{i} \beta_{\nu}+\left(\bar{\nabla}_{i} \alpha+V^{\nu} \bar{\nabla}_{i} \beta_{\nu}\right)$ and $g^{\mu \nu}=-\tau^{\mu} \tau^{\nu}+\bar{g}^{\mu \nu}$, then the integrals over $\mathcal{D}_{t}$ from the above calculation are:

$$
\begin{array}{r}
\int_{\mathcal{D}_{t}} \frac{1}{2} \gamma^{i j}\left(g^{\mu \nu} Q\left(\bar{\nabla}_{i} \beta_{\mu}, \bar{\nabla}_{j} \beta_{\nu}\right)+Q\left(\bar{\nabla}_{i} \alpha, \bar{\nabla}_{j} \alpha\right) \kappa\right)\left(-V^{\tau}\right)-Q\left(\bar{\nabla}_{i} \alpha, \bar{\nabla}_{j} \beta_{\mu}\right) \tau^{\mu} d V_{\bar{g}} \\
=\int_{\mathcal{D}_{t}} \frac{1}{2} \gamma^{i j}\left(\left(-\tau^{\mu} \tau^{\nu} Q\left(\bar{\nabla}_{i} \beta_{\mu}, \bar{\nabla}_{j} \beta_{\nu}\right)+\bar{g}^{\mu \nu} Q\left(\bar{\nabla}_{i} \beta_{\mu}, \bar{\nabla}_{j} \beta_{\nu}\right)+Q\left(\bar{\nabla}_{i} \alpha, \bar{\nabla}_{j} \alpha\right) \kappa\right)\left(-V^{\tau}\right)\right. \\
\left.+Q\left(\bar{\nabla}_{i} \beta_{\nu}, \bar{\nabla}_{j} \beta_{\mu}\right) V^{\nu} \tau^{\mu}-Q\left(\bar{\nabla}_{i} \alpha+V^{\nu} \bar{\nabla}_{i} \beta_{\nu}, \bar{\nabla}_{j} \beta_{\mu}\right) \tau^{\mu}\right) d V_{\bar{g}}
\end{array}
$$

Next we write:

$$
-Q\left(\bar{\nabla}_{i} \beta_{\nu}, \bar{\nabla}_{j} \beta_{\mu}\right) V^{\nu} \tau^{\mu}=Q\left(\bar{\nabla}_{i} \beta_{\nu}, \bar{\nabla}_{j} \beta_{\mu}\right) \tau^{\nu} \tau^{\mu} V^{\tau}-Q\left(\bar{\nabla}_{i} \beta_{\nu}, \bar{\nabla}_{j} \beta_{\mu}\right) \bar{V}^{\nu} \tau^{\mu}
$$

and so the above is:

$$
\begin{aligned}
\frac{1}{2} \int_{\mathcal{D}_{t}} \gamma^{i j}\left(\tau^{\mu} \tau^{\nu} Q\left(\bar{\nabla}_{i} \beta_{\mu}, \bar{\nabla}_{j} \beta_{\nu}\right)+\bar{g}^{\mu \nu} Q\left(\bar{\nabla}_{i} \beta_{\mu}, \bar{\nabla}_{j} \beta_{\nu}\right)+Q\left(\bar{\nabla}_{i} \alpha, \bar{\nabla}_{j} \alpha\right) \kappa\right)\left(-V^{\tau}\right) d V_{\bar{g}} \\
+\int_{\mathcal{D}_{t}} \gamma^{i j} Q\left(\bar{\nabla}_{i} \beta_{\nu}, \bar{\nabla}_{j} \beta_{\mu}\right) \bar{V}^{\nu} \tau^{\mu} d V_{\bar{g}}+\int_{\mathcal{D}_{t}} \gamma^{i j} Q\left(\bar{\nabla}_{i} \alpha+V^{\nu} \bar{\nabla}_{i} \beta_{\nu}, \bar{\nabla}_{j} \beta_{\mu}\right) \tau^{\mu} d V_{\bar{g}}
\end{aligned}
$$

Now we note that:

$$
\left|Q\left(\bar{\nabla}_{i} \beta_{\nu}, \bar{\nabla}_{j} \beta_{\mu}\right) \bar{V}^{\nu} \tau^{\mu}\right| \leq \frac{1}{2}|\bar{V}|\left(\tau^{\mu} \tau^{\nu} Q\left(\bar{\nabla}_{i} \beta_{\mu}, \bar{\nabla}_{j} \beta_{\nu}\right)+\bar{g}^{\mu \nu} Q\left(\bar{\nabla}_{i} \beta_{\mu}, \bar{\nabla}_{j} \beta_{\nu}\right)\right),
$$

so the sum of the terms in the first line of (6.21) with the first term on the second line is bounded below by:

$$
\begin{aligned}
& \frac{1}{2} \int_{\mathcal{D}_{t}} \gamma^{i j}\left(\tau^{\mu} \tau^{\nu} Q\left(\bar{\nabla}_{i} \beta_{\mu}, \bar{\nabla}_{j} \beta_{\nu}\right)\left(-V^{\tau}-|\bar{V}|\right)+\bar{g}^{\mu \nu} Q\left(\bar{\nabla}_{i} \beta_{\mu}, \bar{\nabla}_{j} \beta_{\nu}\right)\left(-V^{\tau}-|\bar{V}|\right)+Q\left(\bar{\nabla}_{i} \alpha, \bar{\nabla}_{j} \alpha\right) \kappa\left(-V^{\tau}\right) d V_{\bar{g}}\right. \\
= & \frac{1}{2} \int_{\mathcal{D}_{t}} \gamma^{i j}\left(\tau^{\mu} \tau^{\nu} Q\left(\bar{\nabla}_{i} \beta_{\mu}, \bar{\nabla}_{j} \beta_{\nu}\right)+\bar{g}^{\mu \nu} Q\left(\bar{\nabla}_{i} \beta_{\mu}, \bar{\nabla}_{j} \beta_{\nu}\right)\right) \frac{\sigma}{|\bar{V}|-V^{\tau}} d V_{\bar{g}}+\int_{\mathcal{D}_{t}} \gamma^{i j} Q\left(\bar{\nabla}_{i} \alpha, \bar{\nabla}_{j} \alpha\right) \kappa\left(-V^{\tau}\right) d V_{\bar{g}},
\end{aligned}
$$

where we have used that $\left(-V^{\tau}-|\bar{V}|\right)\left(-V^{\tau}+|\bar{V}|\right)=\sigma$. This plus the first term from (6.19) is $\mathcal{E}(t)$.

The remaining terms involving integrals over $\mathcal{D}$ and $\Lambda$ are the error terms:

$$
\begin{aligned}
\int_{\mathcal{D}} \gamma^{i j}\left(g^{\mu \nu} Q\left(\nabla_{V} \bar{\nabla}_{i} \beta_{\mu}+\nabla_{\mu} \bar{\nabla}_{i} \alpha, \bar{\nabla}_{j} \beta_{\nu}\right)-Q\left(\bar{\nabla}_{i} \alpha, \operatorname{div} \bar{\nabla}_{j} \beta\right.\right. & \left.\left.+\kappa \nabla_{V} \bar{\nabla}_{j} \alpha\right)-Q\left(\bar{\nabla}_{i} \alpha+V^{\nu} \bar{\nabla}_{i} \beta_{\nu}, \bar{\nabla}_{j} \beta_{\mu}\right) \tau^{\mu}\right) d V_{g} \\
& +\int_{\Lambda} Q\left(\bar{\nabla}_{i} \alpha, N^{\mu} \bar{\nabla}_{j} \beta_{\mu}-\nu \nabla_{V} \bar{\nabla}_{j} \alpha\right) d V_{\gamma}, \quad(6.24)
\end{aligned}
$$

and the terms where derivatives fall on the coefficients:

$$
\begin{aligned}
\int_{\mathcal{D}} \nabla_{\mu}\left(\gamma^{i j} \gamma^{I J}\right) g^{\mu \nu}\left(\bar{\nabla}_{i} \alpha_{I}\right)\left(\bar{\nabla}_{j} \beta_{J \nu}\right)+\nabla_{\mu^{\prime}}\left(\gamma^{i j} \gamma^{I J} V^{\mu^{\prime}}\right) g^{\mu \nu} \bar{\nabla}_{i} \beta_{I \mu} \bar{\nabla}_{j} \beta_{J \nu}+\nabla_{\mu}\left(\gamma^{i j} \gamma^{I J} \kappa V^{\mu}\right)\left(\bar{\nabla}_{i} \alpha_{I}\right)\left(\bar{\nabla}_{j} \alpha_{J}\right) d V_{\gamma} \\
+\int_{\Lambda} \nabla_{\mu}\left(\gamma^{i j} \gamma^{I J} V^{\mu}(-\nu)\right)\left(\bar{\nabla}_{i} \alpha_{I}\right)\left(\bar{\nabla}_{j} \alpha_{J}\right) d V_{g}
\end{aligned}
$$

The terms in (6.24) are bounded by the right-hand side of (6.10). To deal with the first term in (6.25), we first write:

$$
\bar{\nabla}_{j} \beta_{J \nu}=-\left(\bar{\nabla}_{j} \beta_{J \tau}\right) \tau_{\nu}+\bar{\nabla}_{j} \bar{\beta}_{J \nu}-\beta_{J \nu^{\prime}} \bar{\nabla}_{j} \tau^{\nu^{\prime}} \tau_{\nu}
$$

where $\beta_{J \tau}=\beta_{J \mu} \tau^{\mu}$. To control the terms resulting from the components $\bar{\beta}$, we use the elliptic estimate (4.6):

$$
\begin{aligned}
& \left|\int_{\mathcal{D}_{t}} \nabla_{\mu}\left(\gamma^{i j} \gamma^{I J}\right) g^{\mu}\left(\bar{\nabla}_{i} \alpha_{I}\right)\left(\bar{\nabla}_{j} \bar{\beta}_{J \nu^{\prime}}\right) d V_{\bar{g}}\right| \\
& \leq C\left(K E+\left(\|\overline{\operatorname{div}} \alpha\|_{L^{2}\left(\mathcal{D}_{t}\right)}+\|\overline{\operatorname{curl}} \alpha\|_{L^{2}\left(\mathcal{D}_{t}\right)}\right)\left(\|\overline{\operatorname{div}} \bar{\beta}\|_{L^{2}\left(\mathcal{D}_{t}\right)}+\|\overline{\operatorname{curl}} \bar{\beta}\|_{L^{2}\left(\mathcal{D}_{t}\right)}\right) .\right.
\end{aligned}
$$

We will deal with the term coming from $\bar{\nabla}_{j} \beta_{J \tau}$ momentarily. 
To deal with the second term in (6.25), we use the same argument to bound the spatial components $\bar{\beta}$ by:

$$
\begin{aligned}
\left|\int_{\mathcal{D}_{t}} \nabla_{\mu^{\prime}}\left(\gamma^{i j} \gamma^{I J} V^{\mu^{\prime}}\right) g^{\mu}\left(\bar{\nabla}_{i} \bar{\beta}_{I \mu}\right)\left(\bar{\nabla}_{j} \bar{\beta}_{J \nu}\right) d V_{\bar{g}}\right| & \\
& \leq C\left(K E+\left(\|\overline{\operatorname{div}} \bar{\beta}\|_{L^{2}\left(\mathcal{D}_{t}\right)}+\|\overline{\operatorname{curl}} \bar{\beta}\|_{L^{2}\left(\mathcal{D}_{t}\right)}\right)^{2} .\right.
\end{aligned}
$$

To control the second term in (6.25), we use the same argument as well as the assumption (6.4) on $\kappa$ :

$$
\begin{aligned}
&\left|\int_{\mathcal{D}_{t}} \nabla_{\mu}\left(\gamma^{i j} \gamma^{I J} \kappa V^{\mu}\right)\left(\bar{\nabla}_{i} \alpha_{I}\right)\left(\bar{\nabla}_{j} \alpha_{J}\right) d V_{\bar{g}}\right| \\
& \leq C\left(\left(C_{1}+K\right) E+\|\overline{\operatorname{div}} \alpha\|_{L^{2}\left(\mathcal{D}_{t}\right)}^{2}+\|\overline{\operatorname{curl}} \alpha\|_{L^{2}\left(\mathcal{D}_{t}\right)}^{2}\right) .
\end{aligned}
$$

For the boundary term in (6.25), we instead use the assumption (6.5) on $\nu$ :

$$
\left|\int_{\mathcal{D}_{t}} \nabla_{\mu}\left(\gamma^{i j} \gamma^{I J} V^{\mu}(-\nu)\right)\left(\bar{\nabla}_{i} \alpha_{I}\right)\left(\bar{\nabla}_{j} \alpha_{J}\right) d V_{\gamma}\right| \leq C\left(C_{2}+1\right) E
$$

Finally, to deal with the terms coming from $\bar{\nabla}_{j} \beta_{J \tau}$, we write:

$$
\nabla_{i} \beta^{\tau}=\frac{1}{2 V^{\tau}}\left(\bar{\nabla}_{i} \alpha+2 \bar{\nabla}_{i} \beta_{\mu} \bar{V}^{\mu}\right)+\left(\bar{\nabla}_{i} \beta^{\tau}-\frac{1}{2 V^{\tau}}\left(\bar{\nabla}_{i} \alpha+2 \bar{\nabla}_{i} \beta_{\mu} \bar{V}^{\mu}\right)\right) .
$$

We can then use the same arguments as above to bound the terms resulting from the terms in the first bracket here, and after multiplying by $V^{\tau}$, the terms in the second bracket here are bounded by the fourth term on the right-hand side of (6.10).

\section{THE HIGHER-ORDER EQUATIONS}

Lemma 6. Suppose that $u$ satisfies (2.35) and that the assumptions (2.20), (2.40), (2.41), (2.43) and (2.44) hold. If $V, \sigma$ satisfy (1.16) and (1.17) then:

$$
\begin{aligned}
& \left|\nabla_{V} \bar{\nabla}^{r} V+\frac{1}{2} \nabla \bar{\nabla}^{r} \sigma\right|+\left|\nabla_{V} \operatorname{curl} \bar{\nabla}^{r-1} V\right| \leq C \sum_{s=1}^{r}\left|\left(\bar{\nabla}^{s} V\right)\left(\bar{\nabla}^{r-s} \nabla V\right)\right|+R \sum_{s=1}^{r-1}\left|\nabla^{s} V\right|, \\
& \left|\frac{1}{2} \bar{\nabla}^{r} \sigma+V^{\mu} \bar{\nabla}^{r} V_{\mu}\right| \leq C \sum_{s=1}^{r}\left|\bar{\nabla}^{s} V^{\mu}\right|\left|\bar{\nabla}^{r-s} V_{\mu}\right|, \\
& \left|\not h\left(\nabla_{V} \bar{\nabla}^{r} \sigma+\left(\bar{\nabla}^{r} V\right) \cdot \nabla \sigma-\bar{\nabla}^{r} \nabla_{V} \sigma\right)\right| \leq C \sum_{s=1}^{r-1}\left|\not \Lambda\left(\left(\bar{\nabla}^{s} V^{\mu}\right)\left(\bar{\nabla}^{r-s} \nabla_{\mu} \sigma\right)\right)\right| .
\end{aligned}
$$

and there is a polynomial $P$ so that:

$\left|\operatorname{div} \bar{\nabla}^{r} V+e^{\prime}(\sigma) \nabla_{V} \bar{\nabla}^{r} \sigma\right|$

$$
\leq C(M) \sum_{s=1}^{r}\left|\bar{\nabla}^{s} V\right|\left|\bar{\nabla}^{r-s} \nabla V\right|+R \sum_{s=0}^{r-1}\left|\nabla^{s} V\right|+P\left(L,|\bar{\nabla} \sigma|, \ldots,\left|\bar{\nabla}^{r-1} \sigma\right|\right) .
$$

For $k+\ell=r-1, k \geq 2$, there is a polynomial $P$ so that:

$$
\begin{aligned}
\left|\bar{\nabla}^{k-2} \bar{\Delta} \nabla_{u}^{\ell} \sigma-\left(\bar{\nabla}^{k-2} \nabla_{u}^{\ell-1} \bar{\Delta} u^{\mu}\right) \nabla_{\mu} \sigma\right| & \\
\leq C\left(\left|\bar{\nabla}^{k-2} \nabla_{u}^{\ell+2} \sigma\right|+\lambda\left|\bar{\nabla}^{k} \nabla_{u}^{\ell} \sigma\right|\right. & +\sum_{s=0}^{r-1}\left|\left(\nabla^{s+1} V\right)\left(\nabla^{r-s+1} V\right)\right| \\
& \left.+P\left(R, L,|\nabla \sigma|, \ldots\left|\nabla^{r-2} \sigma\right|,|\nabla u|, \ldots,\left|\nabla^{r-2} u\right|\right)\right) .
\end{aligned}
$$


Proof. By (1.16) $),\left(\operatorname{curl} \nabla_{V} V\right)_{\mu \nu}=0$. Therefore, writing $\nabla_{\mu}\left(V^{\nu^{\prime}} \nabla_{\nu^{\prime}}\right)=\left(\nabla_{\mu} V^{\nu^{\prime}}\right) \nabla_{\nu^{\prime}}+V^{\nu^{\prime}}$, we have:

$$
\left(\nabla_{V} \operatorname{curl} V\right)_{\mu \nu}=\nabla_{\mu} V^{\nu^{\prime}} \nabla_{\nu^{\prime}} V_{\nu}-\nabla_{\nu} V^{\nu^{\prime}} \nabla_{\nu^{\prime}} V_{\mu} .
$$

The estimates (7.1) and (7.3) then follow from the product rule and the bound (2.20) for the Riemann tensor. For (7.2) we additionally use that $\sigma=-V^{\mu} V_{\mu}$.

To get (7.4), we can argue as in the proof of $(\underline{\mathrm{B} .22})$ to get:

$$
\left|\nabla^{r}\left(e^{\prime}(\sigma) \nabla_{V} \sigma\right)-e^{\prime}(\sigma) \nabla^{r} \nabla_{V} \sigma\right| \leq P\left(L,|\bar{\nabla} \sigma|, \ldots,\left|\bar{\nabla}^{r-1} \sigma\right|\right)
$$

The estimate (7.5) is similar and uses the equation (5.48) as well as (B.10).

We will also need estimates for $\overline{\operatorname{div}} V$ and $\overline{\operatorname{curl}} V$ in terms of $\operatorname{div} V$ and $\operatorname{curl} V$ :

Lemma 7. Suppose that (2.35) holds and that $V, \sigma$ satisfy (1.16) and (1.17). Then:

$$
\begin{gathered}
\left|\overline{\operatorname{div}} \bar{\nabla}^{r-1} V\right| \leq\left|\bar{\nabla}^{r-1} \nabla_{V} e(\sigma)\right|+C(M, L)\left(\left|\nabla_{V} \bar{\nabla}^{r-1} V\right|+\lambda\left|\bar{\nabla}^{r} V\right|+P\left(\left|\nabla^{r-2} V\right|, R\right) \sum_{k=1}^{r}\left|\nabla^{k-1} V\right|\right), \\
\left|\overline{\operatorname{curl}} \bar{\nabla}^{r-1} V\right| \leq\left|\bar{\nabla}^{r-1} \operatorname{curl} V\right|+C(M, L)\left(\left|\nabla_{V} \bar{\nabla}^{r-1} V\right|+\lambda\left|\bar{\nabla}^{r} V\right|+P\left(\left|\nabla^{r-2} V\right|, R\right) \sum_{k=1}^{r}\left|\nabla^{k-1} V\right|\right) .
\end{gathered}
$$

Proof. By the definition (2.8), we have:

$$
\nabla_{\mu} V^{\nu}=\bar{\nabla}_{\mu} V^{\nu}+\tau_{\mu} \tau_{\nu} \tau^{\mu^{\prime}} \tau^{\nu^{\prime}} \nabla_{\mu^{\prime}} V^{\nu^{\prime}}-\left(\tau_{\mu} \tau^{\mu^{\prime}} \bar{\Pi}_{\nu}^{\nu^{\prime}}+\tau_{\nu} \tau^{\nu^{\prime}} \bar{\Pi}_{\mu}^{\mu^{\prime}}\right) \nabla_{\mu^{\prime}} V^{\nu^{\prime}}
$$

and taking the trace and anti-symmetric part of this identity shows that:

$$
\begin{aligned}
\overline{\operatorname{div}} V & =\operatorname{div} V+\tau_{\mu} \nabla_{\tau} V^{\mu}, \\
\overline{\operatorname{curl}} V_{\mu \nu} & =\operatorname{curl} V_{\mu \nu}+(A V)_{\mu \nu}+(B V)_{\mu \nu},
\end{aligned}
$$

where $A V$ is the anti-symmetric part of:

$$
\tau_{\mu} \nabla_{\tau} \bar{V}^{\nu}+\tau^{\nu} \bar{\nabla}_{\mu} V_{\tau}
$$

and $B V$ is the anti-symmetric part of:

$$
\tau_{\mu}\left(\nabla_{\tau} \bar{\Pi}_{\nu}^{\nu^{\prime}}\right) V_{\nu^{\prime}}+\tau_{\nu} \bar{\Pi}_{\mu}^{\nu^{\prime}}\left(\nabla_{\mu^{\prime}} \tau^{\nu^{\prime}}\right) V_{\nu^{\prime}}
$$

To get (17.8) and (7.9), by (2.20), it suffices to control $\bar{\nabla}^{r-1} \nabla_{\tau} V$. To do this, we write $\nabla_{\tau}=\frac{1}{V^{\tau}}\left(\nabla_{V}-\bar{V}^{\mu} \nabla_{\mu}\right)$ and we have:

$$
\left|\bar{\nabla}^{r-1} \nabla_{\tau} V\right| \leq \frac{1}{\left|u^{\tau}\right|}\left(\left|\bar{\nabla}^{r-1} \nabla_{u} V\right|+|\bar{u}|\left|\bar{\nabla}^{r} V\right|+\left|\bar{\nabla}^{r-1} u\right||\nabla V|\right)+P\left(\mid \nabla^{r-3} u, R\right)\left|\nabla^{r-1} V\right| .
$$

By (2.35), this implies (7.8) and (7.9).

\section{EnERGy ESTimates FOR SOlutions of Euler's EQUATIONS}

The energies for Euler's equation are:

$$
\begin{gathered}
\mathcal{E}^{k, \ell}(t)=\frac{1}{2} \int_{\mathcal{D}_{t}}\left(\tau_{\mu} \tau_{\nu}+\bar{g}_{\mu \nu}\right) Q\left(\bar{\nabla}^{k} \nabla_{u}^{\ell} V^{\mu}, \bar{\nabla}^{k} \nabla_{u}^{\ell} V^{\nu}\right) \frac{\sqrt{\sigma}}{|\bar{u}|-u^{\tau}} \\
+\frac{1}{4} \int_{\mathcal{D}_{t}} e^{\prime}(\sigma) Q\left(\bar{\nabla}^{k} \nabla_{u}^{\ell} \sigma, \bar{\nabla}^{k} \nabla_{u}^{\ell} \sigma\right)\left(-V^{\tau}\right) d V_{\bar{g}} \\
+\frac{1}{4} \int_{\partial \mathcal{D}_{t}} Q\left(\bar{\nabla}^{k} \nabla_{u}^{\ell} \sigma, \bar{\nabla}^{k} \nabla_{u}^{\ell} \sigma\right) \frac{\left(-V^{\tau}\right)}{\left|\nabla_{N} \sigma\right|} d V_{\gamma}, \\
\mathcal{K}^{r}(t)=\int_{\mathcal{D}_{t}}\left|\operatorname{curl} \bar{\nabla}^{r-1} V\right|^{2} d V_{\bar{g}}
\end{gathered}
$$


and the energies for the wave equation (5.13) satisfied by $\sigma$ are:

$$
\mathcal{W}^{r}(t)=\int_{\mathcal{D}_{t}}\left|\nabla_{u}^{r+1} \sigma\right|^{2}+\Pi^{\mu \nu}\left(\nabla_{\mu} \nabla_{u}^{r} \sigma\right)\left(\nabla_{\nu} \nabla_{u}^{r} \sigma\right) d V_{\bar{g}}+\int_{\mathcal{D}_{t}}\left|\nabla_{u}^{r+1} \sigma\right|^{2}\left(\eta^{-2}-1\right)\left(-u^{\tau}\right) d V_{\bar{g}} .
$$

We define:

$$
\mathcal{E}^{r}(t)=\sum_{k+\ell \leq r} \mathcal{E}^{k, \ell}(t)+\mathcal{K}^{r}(t)+\mathcal{W}^{r}(t)
$$

Theorem 2. Suppose that (2.20), (2.40), (2.41) and (2.44) hold for $N \geq r+1$ and that the a priori assumptions (2.35), (2.46), (2.47) and (2.49) hold. For $r \geq 0$, there is a continuous function $C=$ $C\left(r, M, \lambda, L, \delta^{-1}, t, R\right)$ and a polynomial so that if $V, \sigma$ satisfy (1.16)-(1.17) and (1.8) $-(1.11$ ) for $0 \leq t \leq T$, then

for $0 \leq t \leq T$.

$$
\mathcal{E}^{r}(t) \leq \mathcal{E}^{r}(0)+C \int_{0}^{t} \mathcal{E}^{r}(s)+P\left(\mathcal{E}^{r-1}(s)\right) d s
$$

In particular, there are continuous functions $C_{r}=C_{r}\left(t, M, \lambda, L, \delta^{-1}, R, \mathcal{E}^{r-1}(0)\right)$ so that:

$$
\mathcal{E}^{r}(t) \leq C_{r} \mathcal{E}^{r}(0) \text {. }
$$

Furthermore, for large enough $r$ we can get back control of the a priori assumptions. Let:

$$
\begin{aligned}
K(t) & =\|\theta(t, \cdot)\|_{L^{\infty}\left(\partial \mathcal{D}_{t}\right)}+\frac{1}{\iota_{0}(t)}, \\
\tilde{\sigma}(t) & =\|\left(\sigma(t, \cdot)^{-1} \|_{L^{\infty}\left(\mathcal{D}_{t}\right)},\right. \\
\delta(t) & =\|\left(\bar{\nabla} p(t, \cdot)^{-1} \|_{L^{\infty}\left(\partial \mathcal{D}_{t}\right)},\right. \\
\lambda(t) & =\left\|\bar{u}(t)\left(u^{\tau}(t)\right)^{-1}\right\|_{L^{\infty}\left(\mathcal{D}_{t}\right)} .
\end{aligned}
$$

Theorem 3. If $r \geq 5, \lambda(0)<\lambda^{*} / 2$ where $\lambda^{*}$ is defined in (13), and the assumptions (2.20), (2.40), (2.41), (2.44) hold with $N \geq r+1$, then there is a continuous function:

$$
\mathcal{T}_{r}=\mathcal{T}_{r}\left(K(0), \lambda(0), \delta(0), L, R, \mathcal{E}^{r}(0), \operatorname{Vol} \mathcal{D}_{t}\right)
$$

so that for $0 \leq T \leq \mathcal{T}_{r}$ :

$$
\mathcal{E}^{r}(t) \leq 2 \mathcal{E}^{r}(0)
$$

As a first step, we show that the energies (8.1) control all derivatives of $V$ and $\sigma$ :

Lemma 8. If $V, \sigma$ satisfy (1.16)-1.19) then there is a polynomial $P$ so that for $k+\ell=r$ :

$$
\left\|\nabla^{r} V\right\|_{L^{2}\left(\mathcal{D}_{t}\right)}^{2}+\left\|\nabla^{r} \sigma\right\|_{L^{2}\left(\mathcal{D}_{t}\right)}^{2} \leq C(\lambda) \mathcal{E}^{r}(t)+P\left(M, \lambda, L, R, \mathcal{E}^{r-1}(t)\right) .
$$

and

$$
\left\|\not h \bar{\nabla}^{r} \sigma\right\|_{L^{2}\left(\partial \mathcal{D}_{t}\right)}^{2} \leq\|\bar{\nabla} \sigma\|_{L^{\infty}\left(\partial \mathcal{D}_{t}\right)} \mathcal{E}^{r}(t) .
$$

Proof. By (B.9), to prove (8.13) it suffices to bound $\left\|\bar{\nabla}^{k} \nabla_{u}^{\ell} V\right\|_{L^{2}\left(\mathcal{D}_{t}\right)}+\left\|\bar{\nabla}^{k} \nabla_{u}^{\ell} \sigma\right\|_{L^{2}\left(\mathcal{D}_{t}\right)}$ when $k+\ell=r$. By (5.56), $\left\|\bar{\nabla}^{k} \nabla_{u}^{\ell} \sigma\right\|_{L^{2}\left(\mathcal{D}_{t}\right)}$ is bounded by the right-hand side of (8.13).

To control $\bar{\nabla}^{k} \nabla_{u}^{\ell} V$, when $\ell=0$ we just use (4.6), the estimates (7.8), (7.9) and the bounds for $\sigma$. For $\ell \geq 1$, we use that $\nabla_{u} V=\frac{1}{2 \sqrt{\sigma}} \nabla \sigma$ and the bounds for $\sigma$. The estimate (8.14) just follows from the boundary term in (8.1).

To close the energies, we will need to show that we control derivatives of $\theta$. As in 6 , the key point is that if the Taylor sign condition holds, then the boundary term in our energy actually gives us the control of $\theta$ that we need:

Proposition 6. If the above assumptions hold:

$$
\left\|\bar{\nabla}^{r-2} \theta\right\|_{L^{2}\left(\partial \mathcal{D}_{t}\right)}^{2} \leq C \mathcal{E}^{r}(t) .
$$

where $C=C\left(M, K, R,\|\theta\|_{L^{\infty}\left(\partial \mathcal{D}_{t}\right)}, \|\left(\bar{\nabla}_{\mathcal{N} \sigma)^{-1}} \|_{L^{\infty}\left(\partial \mathcal{D}_{t}\right)}, \operatorname{Vol}\left(\mathcal{D}_{t}\right), \mathcal{E}^{r-1}(t)\right)\right.$.

Proof. Because $\left|\bar{\nabla}_{\mathcal{N} \sigma}\right| \geq \delta>0$, this follows from (4.24). 
The last ingredient we will need is an estimate for $\not / \bar{\nabla}^{r} \nabla_{V} \sigma$ on the boundary:

Lemma 9. Under the above hypotheses:

$$
\left\|\not h \bar{\nabla}^{r} \nabla_{V} \sigma(t)\right\|_{L^{2}\left(\partial \mathcal{D}_{t}\right)}^{2}+\left\|\bar{\nabla}^{r-1} \nabla_{V} \sigma(t)\right\|_{L^{2}\left(\partial \mathcal{D}_{t}\right)}^{2}+\left\|\bar{\nabla}^{r} \nabla_{V} \sigma(t)\right\|_{L^{2}\left(\mathcal{D}_{t}\right)}^{2} \leq C \mathcal{E}^{r}(t)
$$

where $C=C\left(M, \lambda, L, K, R, \mathcal{E}^{0}(t), \ldots, \mathcal{E}^{r-1}(t)\right)$.

Proof. Adding (4.22) and (4.19) and using (4.25)-(4.26) to deal with the lower order terms shows that for any $\delta>0$, the left-hand side of (8.16) is bounded by:

$$
\begin{aligned}
C\left(R,\|\theta\|_{L^{\infty}\left(\partial \mathcal{D}_{t}\right)},\|\theta\|_{H^{r-3}\left(\partial \mathcal{D}_{t}\right)}\right)\left(\delta\left\|\not \mid \overline{\nabla^{r}} \nabla_{V} \sigma\right\|_{L^{2}\left(\partial \mathcal{D}_{t}\right)}\right. & +\left\|\bar{\Delta} \nabla_{V} \sigma\right\|_{H^{r-2}\left(\mathcal{D}_{t}\right)} \\
& \left.+\left\|\bar{\nabla}^{r-2} \theta\right\|_{L^{2}\left(\partial \mathcal{D}_{t}\right)}\left\|\bar{\nabla}_{\mathcal{N}} \nabla_{V} \sigma\right\|_{L^{\infty}\left(\partial \mathcal{D}_{t}\right)}\right) .
\end{aligned}
$$

To bound $\left\|\bar{\Delta} \nabla_{V} \sigma\right\|_{H^{r-2}\left(\mathcal{D}_{t}\right)}$, we use the equation (5.48) and (5.56):

$$
\left\|\bar{\Delta} \nabla_{V} \sigma\right\|_{H^{r-2}\left(\mathcal{D}_{t}\right)} \leq C\left(\mathcal{W}^{r}(t)+P\left(\|\sigma\|_{r},\|V\|_{r-1}\right)\right),
$$

where $C=C\left(M, \lambda, L, K, \operatorname{Vol}\left(\mathcal{D}_{t}\right),\|\theta\|_{H^{r-2}\left(\partial \mathcal{D}_{t}\right)}\right)$. Using (8.13) and (8.15) and taking $\delta$ sufficiently small, we have (8.16).

Proof of Theorem 2. By theorem 5.55 and the estimates (8.13) and (8.14), $\mathcal{W}^{r}(t)$ is bounded by the righthand side of (8.5).

We first show how to control $\mathcal{E}^{r, 0}$ and $\mathcal{K}^{r}$ and then we sketch the argument for $\mathcal{E}^{k, \ell}$ when $\ell \geq 1$. Using (7.1) it is straightforward to prove:

$$
\mathcal{K}^{r}(t) \leq \mathcal{K}^{r}(0)+C(M, R) \int_{0}^{t}\left\|\nabla^{r} V(s)\right\|_{L^{2}\left(\mathcal{D}_{t}\right)} \sqrt{\mathcal{K}^{r}(s)} d s,
$$

and this is bounded by the right-hand side of (8.5).

To bound $\mathcal{E}^{r, 0}(t)$, we set $\beta=\bar{\nabla}^{r-1} V, \alpha=\frac{1}{2} \bar{\nabla}^{r-1} \sigma, \nu=-\bar{\nabla} \sigma$ and $\kappa=2 e^{\prime}(\sigma)$, and apply (6.10):

$$
\mathcal{E}^{r, 0}(t)-\mathcal{E}^{r, 0}(0) \leq C \int_{0}^{t} \sqrt{\mathcal{E}^{r, 0}(s)}\left(\sqrt{\mathcal{R}_{1}(s)}+\sqrt{\mathcal{R}_{2}(s)}+\sqrt{\mathcal{R}_{3}(s)}\right) d s
$$

where

$$
\begin{aligned}
& \mathcal{R}_{1}(t)=\left\|\nabla_{v} \bar{\nabla}^{r} V+\frac{1}{2} \nabla \bar{\nabla}^{r} \sigma\right\|_{L^{2}\left(\mathcal{D}_{t}\right)}^{2}+\left\|\operatorname{div} \bar{\nabla}^{r} V+e^{\prime}(\sigma) \nabla_{V} \bar{\nabla}^{r} \sigma\right\|_{L^{2}\left(\mathcal{D}_{t}\right)}^{2}, \\
& \mathcal{R}_{2}(t)=\left\|\mathbb{I}\left(N^{\mu} \bar{\nabla}^{r} V_{\mu}-(\nabla \sigma)^{-1} \bar{\nabla}^{r} \sigma\right)\right\|_{L^{2}\left(\partial \mathcal{D}_{t}\right)}^{2},
\end{aligned}
$$

and

$$
\begin{aligned}
\mathcal{R}_{3}(t)=\left(C_{1}+C_{2}+K\right) \mathcal{E}^{r, 0}+\left\|\frac{1}{2} \bar{\nabla}^{r} \sigma+V^{\mu} \bar{\nabla}^{r} V_{\mu}\right\|_{L^{2}\left(\mathcal{D}_{t}\right)}^{2}+\left\|\bar{\Delta} \bar{\nabla}^{r-1} \sigma\right\|_{L^{2}\left(\mathcal{D}_{t}\right)} \\
+\left\|\overline{\operatorname{div}} \bar{\nabla}^{r-1} V\right\|_{L^{2}\left(\mathcal{D}_{t}\right)}+\left\|\overline{\operatorname{curl}} \bar{\nabla}^{r-1} V\right\|_{L^{2}\left(\mathcal{D}_{t}\right)}
\end{aligned}
$$

By (7.1), (7.4), (7.8), (7.9) and (8.13), $\sqrt{\mathcal{R}_{1}}+\sqrt{\mathcal{R}_{3}}$ is bounded by the right-hand side of (8.5).

By (7.3) and the estimate (8.16), to control $\mathcal{R}_{2}(t)$ it is enough to bound:

$$
\left\|\not \mid\left(\left(\bar{\nabla}^{s} V^{\mu}\right)\left(\nabla_{\mu} \bar{\nabla}^{r-s} \sigma\right)\right)\right\|_{L^{2}\left(\partial \mathcal{D}_{t}\right)}
$$

for $s=1, \ldots, r-1$. As in 6 , each of these terms is clearly lower order and we could use the elliptic estimates (4.8) to control each of these. However this would lead to estimates which are not linear to highest order. Instead, it is more efficient to use the interpolation estimate (A.4) which would apply except that one of the derivatives on $\sigma$ is not tangential. See the discussion after equation (7.21) in [6] for how to deal with the case that the derivative $\nabla_{\mu}$ is purely spatial. For the component of $\nabla_{\mu}$ parallel to $\tau_{\mu}$, we use (2.37) and it suffices to control:

$$
\left\|\not \mid\left(\left(\bar{\nabla}^{s} V^{\mu}\right)\left(\bar{V}^{\nu} \nabla_{\nu} \bar{\nabla}^{r-s} \sigma\right)\right)\right\|_{L^{2}\left(\partial \mathcal{D}_{t}\right)}, \quad \quad\left\|\not \Lambda\left(\left(\bar{\nabla}^{s} V^{\mu}\right)\left(\nabla_{V} \bar{\nabla}^{r-s} \sigma\right)\right)\right\|_{L^{2}\left(\partial \mathcal{D}_{t}\right)} .
$$

The first term just involves spatial derivatives and so can be dealt with by interpolation as above, and the second term is lower order by (1.19) and the estimate (4.22). 
To get control of $\mathcal{E}^{k, \ell}$ for $\ell \geq 1$, we note that because $\nabla_{u}^{\ell} \sigma=0$ for any $\ell \geq 1$, the estimate (4.22) tells us that the boundary term in $\mathcal{E}^{k, \ell}$ is actually below top order. We also have $\bar{\nabla}^{k} \nabla_{u}^{\ell} V^{\mu}=-\frac{1}{2} \bar{\nabla}^{k} \nabla_{u}^{\ell-1}\left(\frac{1}{\sqrt{\sigma}} \nabla^{\mu} \sigma\right)$, so by (2.43) and (5.56), this can be bounded by the right-hand side of (8.5).

Proof of Theorem 3. The proof is essentially the same as the proof of Lemma 7.6 of [6]. The point is that by the Sobolev inequalities (A.7) and (A.9) and the formula (1.43), there are continuous functions $F_{j}$ so that if $r_{0} \geq 3 / 2+2$ :

$$
\begin{aligned}
\|\nabla u\|_{L^{\infty}\left(\mathcal{D}_{t}\right)}+\|\nabla \sigma\|_{L^{\infty}\left(\mathcal{D}_{t}\right)} & \leq F_{1}\left(K, \mathcal{E}_{r}, r\right), \\
\left\|\nabla^{2} \sigma\right\|_{L^{\infty}\left(\partial \mathcal{D}_{t}\right)} & \leq F_{2}\left(K, \mathcal{E}_{r}, r\right), \\
\|\theta\|_{L^{\infty}\left(\partial \mathcal{D}_{t}\right)} & \leq F_{4}\left(K, \mathcal{E}_{r}, r\right), \\
\left\|\nabla \nabla_{V} \sigma\right\|_{L^{\infty}\left(\partial \mathcal{D}_{t}\right)} & \leq F_{4}\left(K, \mathcal{E}_{r}, r\right) .
\end{aligned}
$$

Arguing as in the proof of Lemmas 7.7-7.9 from [6] and using the above estimates gives (8.12).

\section{ACKNOWLEDGEMENTS}

The author would like to thank his advisor Hans Lindblad for many lengthy and helpful discussions.

\section{Appendix A. Interpolation and Sobolev inequalities}

The results of this section are well-known. However, what is important here is that the constants in the below inequalities can all be bounded in terms of the injectivity radius. The proofs of these theorems with these constants appear in the appendix to [6] when $R m=0$, and they carry over to the general case without change.

A.1. Interpolation inequalities. We will require interpolation inequalities both on $\partial \Omega$ and $\Omega$.

Lemma 10. Suppose that:

$$
\frac{m}{s}=\frac{k}{p}+\frac{m-k}{q}, 2 \leq p \leq s \leq q \leq \infty,
$$

and let $a=k / m$. Then there is a constant $C$ depending only on $m$ so that for any $(0, r)$ tensor $\alpha$ :

$$
\left\|\nabla^{k} \alpha\right\|_{L^{s}(\partial \Omega)} \leq C\|\alpha\|_{L^{q}(\partial \Omega)}^{1-a}\left\|\nabla^{m} \alpha\right\|_{L^{p}(\partial \Omega)}^{a} .
$$

In addition, if $\iota_{0} \geq \frac{1}{K}$, then:

$$
\sum_{j=0}^{k}\left\|\bar{\nabla}^{j} \alpha\right\|_{L^{s}(\Omega)} \leq C\|\alpha\|_{L^{q}(\Omega)}^{1-a}\left(\sum_{i=0}^{m}\left\|\bar{\nabla}^{i} \alpha\right\|_{L^{p}(\Omega)} K^{m-i}\right)^{a} .
$$

In particular, if $\ell+m=k$ then:

$$
\left\|\not \nabla^{\ell} \alpha \nabla^{m} \beta\right\|_{L^{2}(\Omega)} \leq C\left(\|\alpha\|_{L^{\infty}(\partial \Omega)}\|\beta\|_{H^{k}(\partial \Omega)}+\|\beta\|_{L^{\infty}(\partial \Omega)}\|\alpha\|_{H^{k}(\partial \Omega)}\right),
$$

and

$$
\left\|\bar{\nabla}^{\ell} \alpha \bar{\nabla}^{m} \beta\right\|_{L^{2}(\Omega)} \leq C(K)\left(\|\alpha\|_{L^{\infty}(\Omega)}\|\beta\|_{H^{k}(\Omega)}+\|\beta\|_{L^{\infty}(\Omega)}\|\alpha\|_{H^{k}(\Omega)}\right) .
$$

Proof. The inequalities (A.2) and (A.3) are (A.4) and (A.12) in 6. The inequalities (A.4) and (A.5) folow from (A.2) and (A.3) by Hölder's inequality; see eg. 29. 


\section{A.2. Sobolev and Poincaré inequalities.}

Lemma 11. Suppose that $1 / \iota_{0} \leq K$. Then for any $(0, r)$-tensor:

$$
\begin{array}{rlrl}
\|\alpha\|_{L^{2 p / 2-k p}(\partial \Omega)} & \leq C(K) \sum_{\ell=0}^{k}\left\|\bar{\nabla}^{\ell} \alpha\right\|_{L^{p}(\partial \Omega)}, & 1 \leq p \leq \frac{2}{k} \\
\|\alpha\|_{L^{\infty}(\partial \Omega)} \leq C(K) \sum_{0 \leq \ell \leq k-1}\left\|\bar{\nabla}^{\ell} \alpha\right\|_{L^{p}(\partial \Omega)}, & k>\frac{2}{p},
\end{array}
$$

and

$$
\begin{aligned}
\|\alpha\|_{L^{3 p / 3-k p}(\Omega)} \leq C(K) \sum_{\ell=0}^{k}\left\|\bar{\nabla}^{\ell} \alpha\right\|_{L^{p}(\Omega)}, & 1 \leq p \leq \frac{3}{k} \\
\|\alpha\|_{L^{\infty}(\Omega)} \leq C(K) \sum_{0 \leq \ell \leq k-1}\left\|\bar{\nabla}^{\ell} \alpha\right\|_{L^{p}(\Omega)}, & k>\frac{3}{p} .
\end{aligned}
$$

Remark. For (A.6) and (A.7) one can instead think of the norm on the left-hand side as being defined in terms of $\gamma^{I J} \alpha_{I} \alpha_{J}$ and replace the derivatives $\bar{\nabla}$ with $\not \nabla$ on the right-hand side.

We will also need the following version of the Poincaré lemma, whose proof is in [6].

Lemma 12. If $q=0$ on $\partial \Omega$ then:

$$
\begin{aligned}
\|q\|_{L^{2}(\Omega)} & \leq C(\operatorname{Vol} \Omega)^{1 / 3}\|\bar{\nabla} q\|_{L^{2}(\Omega)}, \\
\|\bar{\nabla} q\|_{L^{2}(\Omega)} & \leq C(\operatorname{Vol} \Omega)^{1 / 6}\|\bar{\Delta} q\|_{L^{2}(\Omega)} .
\end{aligned}
$$

APPEndix B. Estimates For REMAinder terms

We begin by collecting the various assumptions we will need about the background spacetime $(M, g)$ and the equation of state:

$$
\begin{aligned}
\sum_{s=1}^{N}\left|\nabla^{s} R m\right|+\left|\nabla^{s} \tau\right| & \leq R, \\
\left|\frac{d^{k}}{d \epsilon^{k}} p(\epsilon)\right| & \leq L_{1}, \text { for } k \leq N, \\
0<L_{2} \leq \eta^{2} & \leq 1, \\
\left|\frac{d^{k}}{d \epsilon^{k}} p(\epsilon)\right| & \leq L_{3}, \text { for } k \leq N,
\end{aligned}
$$

for some large $N$, and we are writing $L=L_{1}+\left(L_{2}\right)^{-1}+L_{3}$. We are also assuming the following bounds for $u$ and $\sigma$ :

$$
\begin{array}{r}
|\nabla u|+|\nabla \sigma| \leq M, \\
\lambda \equiv \frac{|\bar{u}|}{|\bar{u}|} \leq \lambda^{*},
\end{array}
$$

for sufficiently small $\lambda^{*}$.

B.1. Estimates for $\nabla_{\tau}$. We need a few simple results to bound time derivatives $\nabla_{\tau}$ in terms of material derivatives $\nabla_{u}$ and $\bar{u} \bar{\nabla}$. By definition:

$$
\nabla_{\tau}=\frac{1}{u^{\tau}}\left(\nabla_{u}-\bar{u}^{\mu} \nabla_{\mu}\right)
$$

The point of this identity is that $\nabla_{u}$ is a tangential derivative and $\bar{u}$ is small by assumption (B.6).

We set:

$$
|X|_{s}=\sum_{k+\ell \leq s}\left|\bar{\nabla}^{k} \nabla_{u}^{\ell} X\right|
$$

Then: 
Lemma 13. Suppose that (B.1) holds with $N \geq r$. There is a $\lambda^{*}=\lambda^{*}(r)$ so that if $u$ satisfies (B.6) with $\lambda<\lambda^{*}$, there is a polynomial $P$ so that:

$$
\left|\nabla^{r} X\right| \leq C(\lambda)\left(|X|_{r}+|u|_{r-1}|X|_{1}+P\left(|u|_{r-2}, R\right)|X|_{r-1}\right)
$$

and for any function $\psi$, if $k+\ell=r-2$ :

$$
\left|\bar{\nabla}^{k} \nabla_{u}^{\ell} \nabla_{\tau}^{2} \psi\right| \leq\left|\bar{\nabla}^{k} \nabla_{u}^{\ell+2} \psi\right|+\lambda^{2}\left|\bar{\nabla}^{k+2} \nabla_{u}^{\ell} \psi\right|+C(\lambda)\left(|u|_{r-1}|\psi|_{1}+P\left(|u|_{r-2}\right)|\psi|_{r-3}\right)
$$

Proof. For $r=1$, (B.9) follows directly from (B.7). For $r \geq 2$, it suffices to bound $\left|\bar{\nabla}^{k} \nabla_{\tau}^{\ell} X\right|$ for $k+\ell=r$, and applying (B.7), we have:

$$
\begin{aligned}
\left|\bar{\nabla}^{k} \nabla_{\tau}^{\ell} X\right| \leq \frac{1}{\left|u^{\tau}\right|^{\ell}}\left(\left|\bar{\nabla}^{k} \nabla_{u}^{\ell} X\right|+|\bar{u}|^{\ell}\left|\bar{\nabla}^{k+\ell} X\right|\right)+\left|\bar{\nabla}^{k} \nabla_{\tau}^{\ell-1} u\right||\nabla X| & \\
& +P\left(\left|\nabla^{r-2} u\right|,\left|u^{\tau}\right|^{-1}, R\right) \sum_{s=0}^{r-1}\left|\nabla^{s-1} X\right| .
\end{aligned}
$$

The result then follows by induction if $\lambda$ is taken sufficiently small.

To get (B.10), we use (B.7) twice:

$$
\left|\bar{\nabla}^{k} \nabla_{u}^{\ell} \nabla_{\tau}^{2} \psi\right| \leq \frac{1}{\left|u^{\tau}\right|^{2}}\left(\left|\bar{\nabla}^{k} \nabla_{u}^{\ell+2} \psi\right|+|\bar{u}|^{2}\left|\bar{\nabla}^{k} \nabla_{u}^{\ell} \bar{\nabla}^{2} \psi\right|\right)+\left|\bar{\nabla}^{k} \nabla_{u}^{\ell} \nabla u\right||\nabla \psi|+P\left(|u|_{r-2}, R\right)|\psi|_{r-1} .
$$

The factor $\left|\bar{\nabla}^{k} \nabla_{u}^{\ell} \nabla u\right|$ can be bounded using (B.9) . We also have:

$$
\left|\bar{\nabla}^{k} \nabla_{u}^{\ell} \bar{\nabla}^{2} \psi\right| \leq\left|\bar{\nabla}^{k+2} \nabla_{u}^{\ell} \psi\right|+\left|\bar{\nabla}^{k} \nabla_{u}^{\ell-1} u\right||\bar{\nabla} \psi|+P\left(|u|_{r-4}, R\right)|\psi|_{r-2},
$$

which implies (B.10).

B.2. Estimates for $\mathcal{F}_{r}, e_{r}, g_{r}$. Recall that we have defined:

$$
\begin{aligned}
\mathcal{F}_{k, \ell} & =-\bar{\nabla}^{k} \nabla_{u}^{\ell}\left(\left(\nabla_{\mu} V^{\nu}\right)\left(\nabla_{\nu} V^{\mu}\right)\right), \\
\mathcal{G}_{k, \ell}(\sigma) & =-\bar{\nabla}^{k} \nabla_{u}^{\ell}\left(\left(\nabla_{u} e(\sigma)+\nabla_{u} e^{\prime}(\sigma)\right) \nabla_{u} \sigma\right), \\
g_{k, \ell}^{1}(\sigma) & =\bar{\nabla}^{k}\left(\bar{\Delta} \nabla_{u}^{\ell} \sigma-\nabla_{u}^{\ell} \bar{\Delta} \sigma\right), \\
g_{k, \ell}^{2}(\sigma) & =\bar{\nabla}^{k} \nabla_{u}^{\ell} \nabla_{\tau}^{2} \sigma-\nabla_{\tau}^{2} \bar{\nabla}^{k} \nabla_{u}^{\ell} \sigma, \\
e_{k, \ell}(\sigma) & =\eta^{-2} \bar{\nabla}^{k} \nabla_{u}^{\ell+2} \sigma-\bar{\nabla}^{k} \nabla_{u}^{\ell}\left(\eta^{-2} \nabla_{u}^{2} \sigma\right) .
\end{aligned}
$$

and that we are writing:

$$
\|f\|_{r}=\sum_{k+\ell \leq r}\left\|\bar{\nabla}^{k} \nabla_{u}^{\ell} f\right\|_{L^{2}(\Omega)}
$$

We first compute:

$$
\left[\nabla_{\mu}, \nabla_{u}\right] f=-\left(\nabla_{\mu} u^{\nu}\right) \nabla_{\nu} f
$$

To bound the above remainder terms, we will need a higher-order version of this, as well as estimates for derivatives of $e(\sigma)$ :

Lemma 14. If $r \geq 5$,(B.1)-(B.4) hold for $N \geq r+1$, and (B.6) holds with sufficiently small $\lambda$, then for any $k+\ell=r$, there is a polynomial $P$ so that:

$$
\left\|\bar{\nabla}^{k} \nabla_{u}^{\ell} f-\nabla_{u}^{\ell} \bar{\nabla}^{k} f+\left(\bar{\nabla}^{k} \nabla^{\ell-1} u^{\mu}\right) \nabla_{\mu} f\right\|_{L^{2}(\Omega)} \leq P\left(\lambda, R,\|u\|_{r-1}\right) \sum_{s=2}^{r-1}\left\|\nabla^{s} f\right\|_{L^{2}(\Omega)},
$$

and a polynomial $P$ so that:

$$
\left\|\bar{\nabla}^{k} \nabla_{u}^{\ell} e^{\prime}(\sigma)-e^{\prime}(\sigma) \bar{\nabla}^{k} \nabla_{u}^{\ell} \sigma\right\|_{L^{2}(\Omega)} \leq P\left(L, \lambda, R,\|\sigma\|_{r-1}\right) .
$$

Proof. We write $D^{j}=\bar{\nabla}^{m} \nabla^{j-m}$ for some $m$. Then, using (2.21), we need to estimate a sum of terms of the form:

$$
u^{m} \cdot\left(D^{r_{1}} u\right)^{p_{1}} \cdots\left(D^{r_{j}} u\right)^{p_{j}}\left(D^{s} f\right)
$$

where $\sum_{i=1}^{j} p_{j}+m=\ell, \sum_{i=1}^{j} r_{i} p_{i}+s=r$ and $s \leq r-1, r_{i} \leq r-2$. If the $r_{i}$ and $s$ are all less than $r-3$ then we get the result by Sobolev embedding and (B.9). When $s=r-2$, then there can't be any $r_{i}$ larger 
than 2 and since $r \geq 5$ we can again apply Sobolev embedding and (B.9). Dealing with the other cases is similar.

To control (B.22), we need to bound a sum of terms of the form:

$$
e^{(m)}(\sigma)(D \sigma)^{p_{1}} \cdots\left(D^{j} \sigma\right)^{p_{j}}
$$

where $m \leq r+1, p_{1}+2 p_{2}+\ldots+j p_{j}=r$ and $j \leq r-1$. If $j \leq r-3$ then we can again use Sobolev embedding to get the result. If $j=r-1$, then note that if $r \geq 3$, this forces $p_{r-1}=1$. Then we have:

$$
\sum_{\ell=1}^{r-2} \ell p_{\ell}+r-1=r,
$$

and so the only option is that $p_{1}=1, p_{\ell}=0$ otherwise, and the result is then clearly bounded by the above. The case $j=r-2$ is similar.

This then implies:

Lemma 15. Let $r \geq 5$. Suppose that the assumptions of the previous lemma hold. For $k+\ell=r$ :

$$
\begin{aligned}
\left\|\mathcal{F}_{k, \ell}\right\|_{L^{2}(\Omega)} & \leq C_{1}\left(\left\|\nabla \nabla^{k} \nabla_{u}^{\ell} V\right\|_{L^{2}(\Omega)}+P\left(\|V\|_{r},\|\sigma\|_{r}, R\right)\right), \\
\left\|g_{k, \ell}^{1}\right\|_{L^{2}(\Omega)}+\left\|g_{k, \ell}^{2}\right\|_{L^{2}(\Omega)}+\left\|\widetilde{g}_{r}\right\|_{L^{2}(\Omega)} & \leq C_{1}\left(\left\|\nabla^{k+\ell+1} u\right\|_{L^{2}(\Omega)}+P\left(\|u\| \|_{r}\right)\|\sigma\|_{r}\right), \\
\left\|e_{k, \ell}\right\|_{L^{2}(\Omega)}+\left\|\mathcal{G}_{k, \ell}\right\|_{L^{2}(\Omega)} & \leq C_{2}\left(\left\|\nabla^{k} \nabla_{u}^{\ell} \sigma\right\|_{L^{2}(\Omega)}+P\left(\|u\|_{r-1},\|\sigma\|_{r-1}\right)\right),
\end{aligned}
$$

where $C_{1}=C_{1}(M, \lambda)$ and $C_{2}=C_{2}(M, \lambda, L)$.

Proof. The estimate (B.26) follows from (B.21) and the estimate (B.27) follows from a straightforward modification of the proof of (B.21).

To prove (B.28), we note that:

$$
\eta^{-2} \bar{\nabla}^{k} \nabla_{u}^{\ell+2} \sigma-\bar{\nabla}^{k} \nabla_{u}^{\ell}\left(\eta^{-2} \nabla_{u}^{2} \sigma\right)=C \eta^{-2-k-\ell} \eta^{(k+\ell)} \bar{\nabla}^{k} \nabla_{u}^{\ell} \sigma+R(\eta, \sigma),
$$

where we are writing $\eta^{(s)}=\frac{d^{s}}{d^{s} \sigma} \eta(\sigma)$ and $R(\eta, \sigma)$ is a sum of terms of the form:

$$
F\left(\eta, \eta^{\prime}, \ldots, \eta^{k+\ell-1}\right)\left(D^{r_{1}} \sigma\right)^{p_{1}} \cdots\left(D^{r_{j}} \sigma\right)^{p_{r}},
$$

where $F$ is bounded if the lower bound in B.3 holds, and where $\sum_{i=1}^{j} r_{i} p_{i}=k+\ell, r_{i} \leq r-1$. As in the previous lemma, all these terms can be bounded by the right-hand side of (B.28) by Sobolev embedding and (B.9). The estimate for $\mathcal{G}_{k, \ell}$ is similar.

\section{REFERENCES}

[1] D. Ebin, "The equations of motion of a perfect fluid with free boundary are not well posed.," Commun. Partial Differ. Equations, vol. 12, pp. 1175-1201, 1987.

[2] D. Christodoulou, "Self-gravitating relativistic fluids: A two-phase model," Arch. Ration. Mech. Anal., vol. 130, no. 4, pp. 343-400, 1995.

[3] S. Wu, "Global wellposedness of the 3-D full water wave problem," Invent. Math., vol. 184, no. 1, pp. 125-220, 2011.

[4] P. Germain, N. Masmoudi, and J. Shatah, "Global solutions for the gravity water waves equation in dimension 3," Ann. Math., vol. 175, no. 2, pp. 691-754, 2012.

[5] A. D. Ionescu and F. Pusateri. Recent advances on the global regularity for irrotational water waves. Philos. Trans. Roy. Soc. A, 376(2111):20170089, 28, 2018.

[6] D. Christodoulou and H. Lindblad, "On the motion of the free surface of a liquid," Commun. Pure Appl. Math., vol. 53, no. 12 , pp. $1536-1602,2000$.

[7] H. Lindblad, "Well-posedness for the motion of an incompressible liquid with free surface boundary," Ann. Math., vol. 162, no. 1, pp. 109-194, 2005.

[8] D. Coutand and S. Shkoller, "Well-posedness of the free-surface incompressible euler equations with or without surface tension," J. Am. Math. Soc., vol. 20, no. 3, pp. 829-930, 2007.

[9] K. H. Nordgren, Well-posedness for the equations of motion of an inviscid, incompressible, self-gravitating fluid with free boundary. PhD thesis, University of California, San Diego, 2008.

[10] H. Lindblad, "Well posedness for the motion of a compressible liquid with free surface boundary," Commun. Math. Phys., vol. 260, no. 2, pp. 319-392, 2005. 
[11] D. Coutand, J. Hole, and S. Shkoller, "Well-Posedness of the Free-Boundary Compressible 3-D Euler Equations with Surface Tension and the Zero Surface Tension Limit," SIAM J. Math. Anal., vol. 45, no. 6, pp. 3690-3767, 2013.

[12] H. Lindblad and C. Luo, "A priori estimates for the compressible euler equations for a liquid with free surface boundary and the incompressible limit," Communications on Pure and Applied Mathematics, vol. 71, pp. 1273-1333, dec 2017.

[13] D. Coutand, H. Lindblad, and S. Shkoller, "A priori estimates for the free-boundary 3d compressible Euler equations in physical vacuum," Commun. Math. Phys., vol. 296, no. 2, pp. 559-587, 2010.

[14] J. Jang and N. Masmoudi, "Well-posedness of Compressible Euler Equations in a Physical Vacuum," Commun. Pure Appl. Math., vol. 68, pp. 61-111, 2015.

[15] T. Makino, "An application of the Nash-Moser theorem to the vacuum boundary problem of gaseous stars," J. Differ. Equ., vol. 262, pp. 803-843, 2017.

[16] M. Hadžić and J. Jang, "A class of global solutions to the Euler-Poisson system," pre-print, 2017. arXiv:1712:0014.

[17] D. Christodoulou, The Formation of Shocks in 3-Dimensional Fluids. Zürich: European Mathematical Society Publishing House, 2007.

[18] D. Christodoulou, "The Shock Development Problem," pre-print, 2017. arXiv:1705.00828

[19] G. Holzegel, S. Klainerman, J. Speck, and W. W.-Y. Wong, "Small-data shock formation in solutions to 3D quasilinear wave equations: An overview," J. Hyperbolic Differ. Equations, vol. 13, no. 01, pp. 1-105, 2016.

[20] T. Oliynyk, "A priori estimates for relativistic liquid bodies," Bull. des Sci. Mathématiques, vol. 141, pp. 105-222, 2017.

[21] T. Oliynyk, "Dynamical relativistic liquid bodies i: constraint propagation," pre-print, 2017. arXiv:1707.08219.

[22] S. Shkoller, J. Speck, and M. Hadžić, "A priori estimates for solutions to the relativistic Euler equations with a moving vacuum boundary," pp. 1-43, 2015.

[23] J. Jang, P. G. LeFloch, and N. Masmoudi, "Lagrangian formulation and a priori estimates for relativistic fluid flows with vacuum," J. Differ. Equ., vol. 260, pp. 5481-5509, 2016.

[24] R. M. Wald, General Relativity. University of Chicago Press, 1984.

[25] L. Rezzolla and O. Zanotti, Relativistic Hydrodynamics. Oxford University Press, 2013.

[26] H. Lindblad and C. Luo, "A priori Estimates for the Compressible Euler Equations for a Liquid with Free Surface Boundary and the Incompressible Limit," pre-print, 2016. arXiv:1611.05278

[27] A. Lichnerowicz, Relativistic hydrodynamics and magnetohydrodynamics. Lectures on the Existence of Solutions. New York: W. A. Benjamin, Inc., 1967.

[28] G. Fournodavlos and V. Schlue, "On "hard stars" in general relativity," pre-print, 2017. arXiv:1710.00754

[29] M. Taylor, Partial differential equations. III, Nonlinear equations. Springer, 2011. 